\title{
Microwave Frequency Demodulation Using two Coupled Optical Resonators with Modulated Refractive Index
}

\author{
Adam Mock $\odot^{*}$ \\ School of Engineering and Technology, Central Michigan University, Mount Pleasant, Michigan 48859, USA
}

(Received 16 October 2020; revised 1 February 2021; accepted 10 February 2021; published 18 March 2021)

\begin{abstract}
Traditional electronic frequency demodulation of a microwave frequency voltage is challenging because it requires complicated phase-locked loops, narrowband filters with fixed passbands, or large footprint local oscillators and mixers. Herein, a different frequency demodulation concept is proposed based on refractive index modulation of two coupled microcavities excited by an optical wave. A frequencymodulated microwave frequency voltage is applied to two photonic crystal microcavities in a spatially odd configuration. The spatially odd perturbation causes coupling between the even and odd supermodes of the coupled-cavity system. It is shown theoretically and verified by finite-difference time-domain simulations how careful choice of the modulation amplitude and frequency can switch the optical output from on to off. As the modulating frequency is detuned from its off value, the optical output switches from off to on. Ultimately, the optical output amplitude is proportional to the frequency deviation of the applied voltage making this device a frequency-modulated-voltage to amplitude-modulated-opticalwave converter. The optical output can be immediately detected and converted to a voltage that would result in a frequency-demodulated voltage signal. Or the optical output can be fed into a larger radioover-fiber optical network. In this case the device presents a compact, low power, and tunable route for multiplexing frequency-modulated voltages with amplitude-modulated optical communication systems. The resulting system requires modest modulation amplitudes and operates at frequencies relevant for modern communication systems. The cavity designs have realistic quality factors that are well within the range of experimental implementation. The role of modulation sidebands in reducing switching contrast is explored, and two methods for mitigating these effects are demonstrated.
\end{abstract}

DOI: 10.1103/PhysRevApplied.15.034056

\section{INTRODUCTION}

The manipulation of eigenmodes in coupled optical resonators is a well-studied component of photonic device design [1-3]. Spatiotemporal modulation of the cavity material offers an additional mechanism by which photonic signal processing capabilities can be implemented [4-6]. For example, spatiotemporal modulation can be used to break time-reversal symmetry resulting in optical isolation [7-9], optical circulation [10], and optical orbital angular momentum generation [11]. On the other hand, the interplay of microwave frequency electronic signals with visible and near-infrared photonic signals underpins many of the capabilities of modern microwave photonic devices $[12,13]$. This work combines these two perspectives to

\footnotetext{
*mock1ap@cmich.edu
}

Published by the American Physical Society under the terms of the Creative Commons Attribution 4.0 International license. Further distribution of this work must maintain attribution to the author(s) and the published article's title, journal citation, and DOI. propose a compact microwave-frequency-controlled photonic switch based on time modulation of two coupled photonic resonators. The overall concept is shown in Fig. 1. A continuous wave $(\mathrm{cw})$ optical carrier is coupled into one cavity resonator of a two-coupled-cavity resonator system. The refractive indices of the two cavities are modulated at a microwave frequency and $180^{\circ}$ out of phase. The amplitude of the resultant output optical wave is then modulated in response to the frequency of the modulating voltage. Control of an optical output intensity by the frequency of an applied voltage is unique to this device topology and is not an effect that can be readily achieved using existing devices such as a standard Mach-Zehnder intensity modulator. Two tandem phase modulators could potentially be used to achieve the same effect though at the expense of larger foot print and higher electrical drive power [14,15].

Frequency modulation (FM) is a classic modulation format recognized for its improved noise immunity over that of amplitude modulation (AM) that comes at the expense of larger bandwidth usage and more complicated modulation and demodulation hardware [16]. However, because FM signals are transmitted at a constant maximized power level, need for power regularization circuitry 


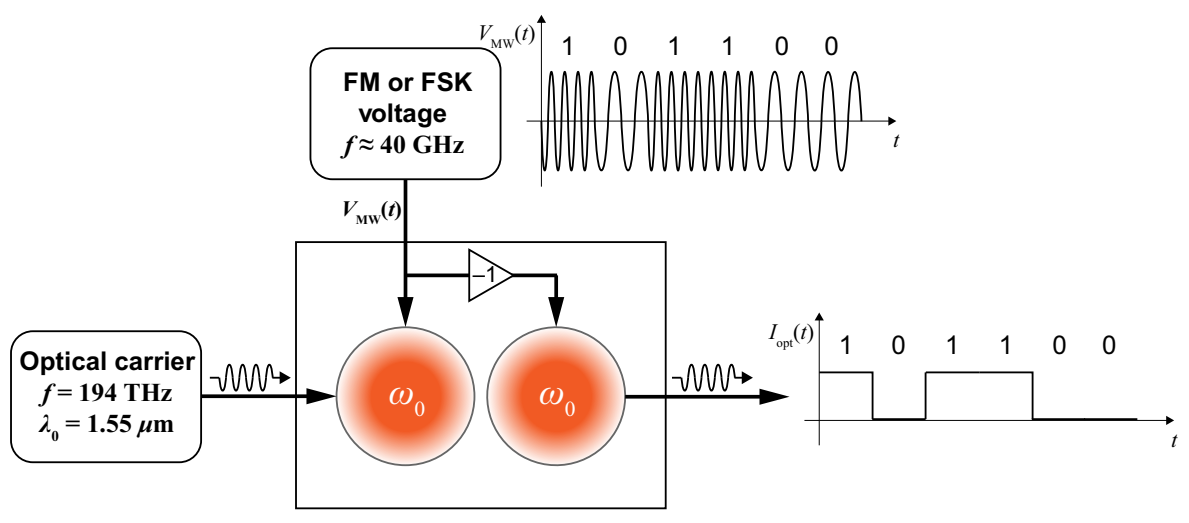

FIG. 1. Depiction of the frequency demodulation concept. An optical carrier is inputted from the left. Two coupled optical resonators are modulated by a frequency-modulated microwave (MW) frequency voltage $V_{\mathrm{MW}}(t)$. The frequency modulation shown is binary FSK. The output optical signal is an amplitude-modulated version of the FSK electrical signal. This optical output can be combined with a larger optical communication system or it can be directly fed to a photodiode for electrical demodulation of the FM signal.

is reduced. Digital signaling is implemented in FM systems by assigning a binary zero to an assigned frequency $f_{0}=f_{c}-f_{d} / 2$ ("space" frequency) and a binary one to a different assigned frequency $f_{1}=f_{c}+f_{d} / 2$ ("mark" frequency), where $f_{c}$ is the carrier frequency and $f_{d}$ is the difference frequency. The minimum difference frequency to maintain orthogonal signal waveforms is $f_{d}=1 /(2 T)$, where $T$ is the signal period, and most traditional demodulation approaches require $f_{d}=1 / T$. Overall, such a scheme is referred to as binary frequency shift keying (FSK) and can be extended to $M$-ary modulation formats as well. Its constant power transmission and enhanced immunity to noise make FSK popular in radio frequency identification (RFID) technologies [17], biomedical implant communication [18], Bluetooth [19], and a variety of home wireless technologies [20]. By the same token, FSK is attractive in visible light digital communication systems to avoid flicker when the carrier wave doubles as a room lighting source $[21,22]$. FSK is also prevalent in radar systems in which ranging and doppler shifts are more accurately measured than in pulsed time-of-flight implementations [23].

The microwave-frequency-controlled switch depicted in Fig. 1 amplitude modulates a cw optical carrier wave in response to an applied FM voltage. If the message signal embedded in the FM voltage is a generic analog signal then the output optical wave is amplitude modulated with the same analog message. If the FM voltage is a FSK signal then the digital information is imparted on the output optical wave in the form of amplitude shift keying (ASK) or on-off keying (OOK). If a photodiode is used immediately to convert the output optical wave into an electrical signal, the proposed switch is a frequency demodulator, producing a demodulated electrical message signal from an electrical frequency-modulated input signal. As discussed later in this work, this electrical-optical-electrical FM demodulator approach is dynamically tunable, so that a single device can be used for different frequency ranges and frequency deviations which makes it advantageous over traditional approaches of FSK demodulation that use narrowband filters or local oscillators with fixed center frequencies. In the case of general FM demodulation it avoids the need for a phase-locked loop (PLL) and works for virtually any microwave frequency. Primary power requirements of the proposed electrical-optical-electrical demodulator are to drive the chip-scale laser source that is similar to that of an electronic PLL or local oscillator.

If the output optical wave is not immediately detected and converted to a voltage, it can be multiplexed with a larger radio-over-fiber optical communication network. In this implementation, the proposed device functionality is attractive as few if any existing compact devices perform the specific task of directly amplitude modulating an optical wave in response to the frequency of an applied voltage. Use of radio over fiber is ubiquitous in wireless communication [24], and microwave and millimeter wave wireless frequencies are a significant and growing component in 5G communication systems [25]. Using conventional demodulation approaches, a FM or FSK electronic signal would need to be converted to an amplitude-modulated electronic signal before it can amplitude modulate an optical carrier to join the larger optical communication network. The proposed device obviates the need for FM to AM conversion in the electrical domain, thereby improving the power efficiency and SNR of the process.

In the following, a coupled mode theory (CMT) model is used to develop the device concept, and results from full-wave finite-difference time-domain (FDTD) electrodynamic simulations confirm device properties derived via CMT. It is shown how the device may be implemented in a one-dimensional (1D) photonic crystal (PC) platform. The 1D PC consists of circular air holes etched into a semiconductor ridge waveguide of width less than $400 \mathrm{~nm}$. The device length is $12.5 \mu \mathrm{m}$. Such a small 
device footprint is advantageous for dense integration and for enhanced light-matter interaction. A refractive index change of $\Delta n=1.48 \times 10^{-3}$ with a modulation frequency $f_{m}=23.8 \mathrm{GHz}$ are shown numerically to produce an on-off ratio of $26.4 \mathrm{~dB}$. These results show that this device design is consistent with well-established experimental capabilities [26-32]. Furthermore, it is shown that there are a wide range of modulation amplitudes and frequencies that may be used to switch the optical output on and off in a single device design. The effect of modulation sidebands is addressed, and effective techniques for their suppression are introduced.

\section{COUPLED MODE THEORY ANALYSIS AND SCATTERING MATRIX}

\section{A. Modes of the two-coupled-cavity system}

Consider the two-cavity system depicted in Fig. 2(a). To obtain design constraints on the proposed system, coupled mode theory analysis is used to find the scattering spectra and their dependence on device parameters [10,33-35]. It is assumed that the fields of the coupled two-cavity system may be constructed from the fields of the isolated resonators according to [36]

$$
|\mathbf{F}(t)\rangle=a_{1}(t)\left|\mathbf{F}_{1}\right\rangle+a_{2}(t)\left|\mathbf{F}_{2}\right\rangle,
$$

where $\left|\mathbf{F}_{1}\right\rangle$ and $\left|\mathbf{F}_{2}\right\rangle$ represent the spatial distributions of the vector fields in resonators 1 and 2, respectively, in the absence of coupling; and $a_{1}(t)$ and $a_{2}(t)$ denote the time-dependent expansion coefficients. Inserting Eq. (1) into Maxwell's equations, and viewing resonator 1 (2) as a small perturbation to resonator 2 (1) results in

$$
\begin{aligned}
& \frac{d a_{1}(t)}{d t}=\left(-i \omega_{0}-\gamma_{0}\right) a_{1}-i \kappa a_{2}, \\
& \frac{d a_{2}(t)}{d t}=\left(-i \omega_{0}-\gamma_{0}\right) a_{2}-i \kappa a_{1} .
\end{aligned}
$$

(a)

(b)
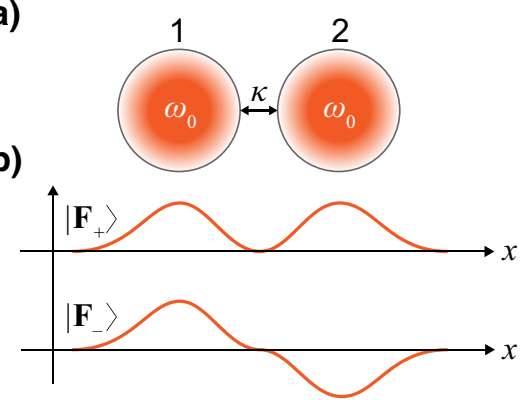

A detailed derivation of Eqs. (2) is provided in Ref. [37]. Letting $a_{k}(t)=a_{k} e^{-i \omega t}$ for $k=1,2$ results in

$$
\begin{aligned}
& -i \omega a_{1}=\left(-i \omega_{0}-\gamma_{0}\right) a_{1}-i \kappa a_{2}, \\
& -i \omega a_{2}=\left(-i \omega_{0}-\gamma_{0}\right) a_{2}-i \kappa a_{1} .
\end{aligned}
$$

These equations can be written as a matrix

$$
-i \omega\left[\begin{array}{l}
a_{1} \\
a_{2}
\end{array}\right]=\left[\begin{array}{cc}
-i \omega_{0}-\gamma_{0} & -i \kappa \\
-i \kappa & -i \omega_{0}-\gamma_{0}
\end{array}\right]\left[\begin{array}{l}
a_{1} \\
a_{2}
\end{array}\right],
$$

from which the eigenfrequencies of the two-resonator system are found to be $\omega_{ \pm}=\omega_{0} \pm \kappa-i \gamma_{0}$. This shows that the nominal resonance frequency $\omega_{0}$ is split to values slightly above and below this value with a similar effect on the loss rate. The corresponding eigenvectors are given by $\mathbf{a}_{+}=[1,1]^{\top} / \sqrt{2}$ and $\mathbf{a}_{-}=[1,-1]^{\top} / \sqrt{2}$, and the full eigenstates are given by

$$
\left|\mathbf{F}_{ \pm}(t)\right\rangle=\frac{1}{\sqrt{2}}\left[\left|\mathbf{F}_{1}\right\rangle \pm\left|\mathbf{F}_{2 q}\right\rangle\right] e^{-i \omega \pm t} .
$$

These eigenstates can be viewed as even (+) and odd (-) supermodes, as depicted in Fig. 2(b).

Figure 2(c) depicts the 1D PC cavity platform in which the two-coupled-cavity system is implemented. The periodic array of air holes creates a photonic bandgap whose center frequency is tuned with hole size and waveguide width. A resonant cavity is formed by removing holes from the array. In this case, three holes are removed to form a cavity with a high quality factor and small footprint [38-41]. A second cavity is coupled to the first one as depicted in the figure. The gray regions denote semiconductor material with refractive index $n=3.1$ consistent with electrorefractive materials suitable for microwave photonics such as silicon and indium phosphide [26,27]. The hole radii are $r=0.3 w$, where $w$ is the width of the waveguide, and the hole spacing is equal to the waveguide

\section{(c)}

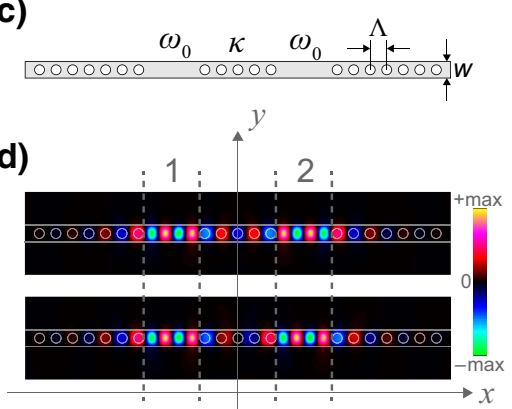

FIG. 2. (a) Two optical resonators defined by a spatially varying electric permittivity $\varepsilon(\mathbf{r})$ whose proximity results in coupling denoted by $\kappa$. The resonators are assumed identical with a nominal resonance frequency $\omega_{0}$. (b) Schematic depiction of the even $\left|\mathbf{F}_{+}\right\rangle$ and odd $\left|\mathbf{F}_{-}\right\rangle$supermodes along the $x$ direction. (c) One-dimensional photonic crystal geometry with two defect cavities. (d) Spatial distribution of the magnetic field $H_{z}(x, y)$ for the even and odd supermodes of the system schematically depicted in (b) calculated using the 2D FDTD method. 
width $\Lambda=w$. A value of $w=0.372 \mu \mathrm{m}$ tunes the device to operate at $\lambda_{0}=1.55 \mu \mathrm{m}$. The coupling rate $\kappa$ may be tuned by the number of air holes between the two cavities; for example, $\kappa$ increases as the cavities are brought closer together. Overall, these geometrical specifications are inline with well-established fabrication capabilities [28,42-44].

It is worth pointing out that the demodulator concept can be implemented in any geometry with waveguides and two coupled cavities having nondegenerate resonances. This includes defect waveguides and cavities in a twodimensional PC lattice $[10,45]$. However, the nanoscale ridge waveguide with a localized 1D PC shown in Fig. 3(b) is easier to fabricate, easier to design, and will likely have lower surface scattering than a full two-dimensional PC lattice while offering the same functionality. Microring resonators are another popular and well-understood cavity system with potential to implement the proposed demodulation concept; however, due to the degenerate (or nearly degenerate) counterpropagating whispering gallery modes in a single resonator, a separate analysis would need to be undertaken to determine the modulation format to obtain similar functionality.

Figure 2(d) shows the spatial distribution of the magnetic field $H_{z}(x, y)$ for the even and odd supermodes of the system depicted in Fig. 2(c) calculated using the 2D FDTD method. The figure shows the field in the $x-y$ plane, where the $\left[E_{x}(x, y), E_{y}(x, y), H_{z}(x, y)\right]$ polarization is used. The even and odd parity along the $x$ direction is apparent along with the mode confinements along $x$ and $y$.

\section{B. Coupling to the two-coupled-cavity system with input and output waveguides}

Light energy will be inserted into the two-resonator system via an input waveguide, and the output energy will be collected via an output waveguide as shown in Fig. 3(a). To account for these energy transfers, Eqs. (3) are modified according to

$$
\begin{aligned}
-i \omega a_{1}(\omega)= & {\left[-i \omega_{0}-\gamma_{0}-\gamma_{c 1}\right] a_{1}(\omega) } \\
& -i \kappa a_{2}(\omega)+d_{1} s_{i 1}(\omega), \\
-i \omega a_{2}(\omega)= & {\left[-i \omega_{0}-\gamma_{0}-\gamma_{c 2}\right] a_{2}(\omega) } \\
& -i \kappa a_{1}(\omega)+d_{2} s_{i 2}(\omega),
\end{aligned}
$$

where $d_{l}$ is the coupling rate from waveguide $l$ and $s_{i l}$ is the input amplitude in waveguide $l[1,10,33-35]$. In this work, waveguides 1 and 2 are the input and output waveguides, respectively, and are symmetrically coupled, so $d_{1}=d_{2} \equiv$ $d$. The waveguide-loaded cavities have a decay rate augmented by $\gamma_{c 1}=\gamma_{c 2} \equiv \gamma_{c}$. The coupling rate and decay rate are related via $2 \gamma_{c}=d^{2}$. In the waveguide-coupledcavity system, the frequency $\omega$ is the excitation frequency, and the amplitudes $a_{l}(\omega)$ are continuous functions of $\omega$. To quantify the energy output, the mode amplitudes are related to the output waves via $s_{r l}(\omega)=-s_{i l}(\omega)+d a_{l}(\omega)$ for $l=1,2$. Figure 3(b) depicts waveguides 1 and 2 implemented in the 1D PC ridge waveguide configuration. Similar to the way $\kappa$ is controlled by the number of air holes between the cavities, the waveguide coupling rate $\gamma_{c}$ is controlled by the number of holes between the uniform ridge waveguide and the defect. Figure 3(c) shows the field distribution when the system is excited at $\omega_{ \pm}$. The relation to the even and odd supermodes is visible, but the amount of energy in the two cavities is no longer equal, which is consistent with theory predictions when the energy is incident from only one side. Figure 3(d) shows the output scattering spectra for waveguides 1 and 2 assuming input in waveguide 1 for typical values of $\omega_{0}, \kappa, d$, and $\gamma_{0}$. The scattering parameters are defined

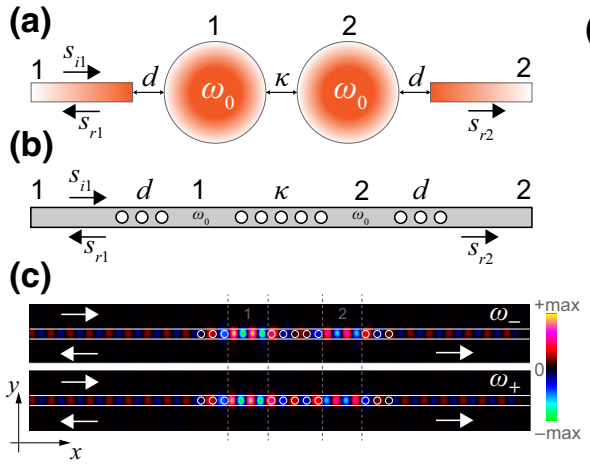

(d)

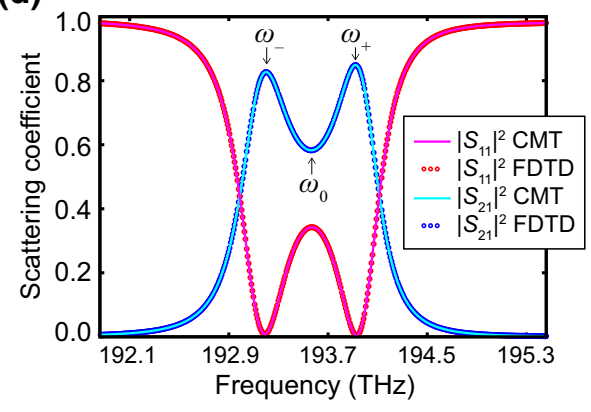

FIG. 3. (a) Diagram showing resonators 1 and 2 coupled to waveguides 1 and 2, respectively. Here $s_{i 1}$ and $s_{r 1}$ denote the incident and reflected wave amplitudes in waveguide 1 , and $s_{r 2}$ denotes the reflected wave amplitude in waveguide 2 . The system is excited from waveguide 1, which makes waveguide 1 the input waveguide and waveguide 2 the output waveguide. (b) Two photonic crystal defect cavities coupled to waveguides. (c) Spatial distribution of the magnetic field $H_{z}(x, y)$ for excitation at $\omega_{-}$and $\omega_{+}$calculated using the two-dimensional finite-difference time-domain method. (d) Scattering spectra for the two-cavity system coupled to waveguides. Solid lines are obtained from CMT analysis. Circles are obtained from 2D FDTD simulation. Cavity parameters are $\omega_{0}=2 \pi(193.5 \mathrm{THz})=$ $2 \pi\left(c_{0} / 1.55 \mu \mathrm{m}\right), \gamma_{0}=1.26 \times 10^{11} \mathrm{~s}^{-1}, \gamma_{c}=1.34 \times 10^{12} \mathrm{~s}^{-1}$, and $\kappa=(2.70+i 0.0114) \times 10^{12} \mathrm{~s}^{-1}$. 


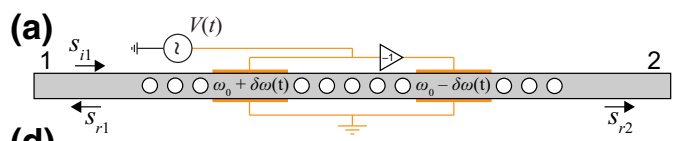

(d)
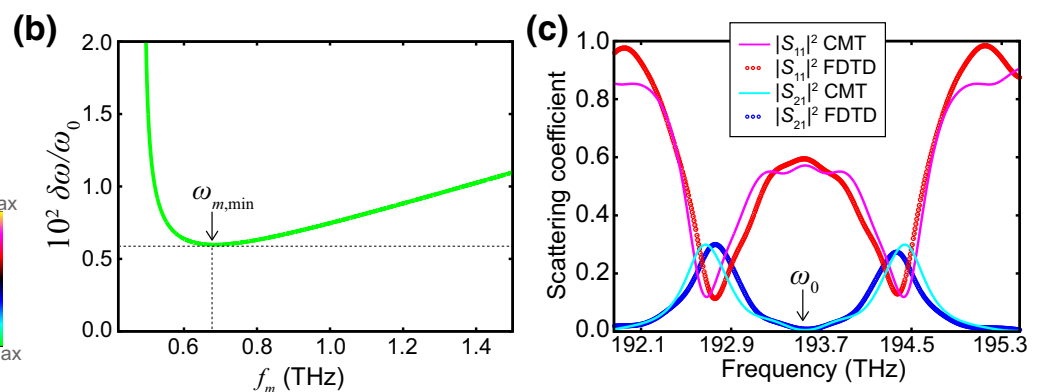

FIG. 4. (a) Depiction of the two resonator system shown in Fig. 3(b) but with metal electrodes shown for modulating the refractive index in the cavity regions. (b) A plot of Eq. (15) for parameters $\gamma=1.461 \times 10^{12} \mathrm{~s}^{-1}$ and $\kappa=2.70 \times 10^{12} \mathrm{~s}^{-1}$. The minimum $\delta \omega$ along with $\omega_{m \text {,min }}$ consistent with Eq. (16) is indicated. (c) Scattering spectra for the modulated two-cavity system coupled to waveguides when the modulation is turned on showing the reduction of $\left|S_{21}\left(\omega_{0}\right)\right|^{2}$. Solid lines are obtained from CMT analysis. Circles are obtained from 2D FDTD simulation. The same parameters as Fig. 3(d) are used in the CMT fit. The modulation parameters are $\Delta n=0.02855$ and $\omega_{m}=2 \pi(683 \mathrm{GHz})$. (d) Spatial distribution of the magnetic field $H_{z}(x, y)$ for excitation at $\omega_{0}$ when the modulation is on (top) and off (bottom) calculated using 2D FDTD.

as $S_{11}(\omega)=s_{r 1}(\omega) / s_{i 1}(\omega)$ and $S_{21}(\omega)=s_{r 2}(\omega) / s_{i 1}(\omega)$. The split resonance shape corresponding to the frequencies $\omega_{ \pm}$is apparent.

\section{Time-modulated two-coupled-cavity system}

Now it is imagined that metallic electrical contacts are connected to the resonators by which a time-dependent voltage $V(\mathbf{r}, t)=V_{m}(\mathbf{r}) \cos \left(\omega_{m} t\right)$ is applied to the material as depicted in Fig. 4(a). In this expression, $\omega_{m}=2 \pi f_{m}$ is the modulation frequency and $V_{m}(\mathbf{r})$ is the spatially dependent amplitude related to the geometry of the contact electrodes. In this way the refractive index changes in proportion to the voltage via the electrorefractive effect according to $n(\mathbf{r}, t)=n_{0}(\mathbf{r})+\Delta n(\mathbf{r}, t)$, where $n_{0}(\mathbf{r})$ is the static two-resonator geometry, and it is assumed that $\Delta n(\mathbf{r}, t)$ depends linearly on the applied voltage $V(\mathbf{r}, t)$ where values up to $|\Delta n| \sim 10^{-2}$ are achievable in silicon and indium phosphide $[26,27]$. The time-dependent change in refractive index produces a corresponding change in the resonance frequency of the isolated resonator $\omega(t)=$ $\omega_{0}+\delta \omega \cos \left(\omega_{m} t\right)$, and the change in resonance frequency is related to the change in refractive index by $\delta \omega=$ $\left(\Delta n / n_{0}\right) \omega_{0}$, where $\Delta n$ and $n_{0}$ are effective indices and depend on the resonance mode of interest.
If one were to modulate both cavities in phase, the resulting effect would be an overall phase modulation of the system producing signal components at $\omega+s \omega_{m}$ for integer $s$. This could be useful if the objective is to phase modulate a carrier wave inputted to the system. However, the demodulation device concept proposed here relies on modulating the cavities with a $180^{\circ}$ phase shift. This is a spatially odd time-dependent perturbation that causes coupling between the even and odd supermodes of the twocavity system. Adding waveguide coupling and the two phase-shifted time-dependent frequency terms to Eqs. (2) results in

$$
\begin{aligned}
\frac{d a_{1}(t)}{d t}= & {\left[-i \omega_{0}-i \delta \omega \cos \left(\omega_{m} t\right)-\gamma\right] a_{1}(t) } \\
& -i \kappa a_{2}(t)+d s_{i 1}(t), \\
\frac{d a_{2}(t)}{d t}= & {\left[-i \omega_{0}+i \delta \omega \cos \left(\omega_{m} t\right)-\gamma\right] a_{2}(t) } \\
& -i \kappa a_{1}(t)+d s_{i 2}(t),
\end{aligned}
$$

where $\gamma \equiv \gamma_{0}+\gamma_{c}$. Equations (7) can be written in matrix form according to

$$
\frac{d}{d t}\left[\begin{array}{l}
a_{1}(t) \\
a_{2}(t)
\end{array}\right]=\left[\begin{array}{cc}
-i \omega_{0}-i \delta \omega \cos \left(\omega_{m} t\right)-\gamma & -i \kappa \\
-i \kappa & -i \omega_{0}+i \delta \omega \cos \left(\omega_{m} t\right)-\gamma
\end{array}\right]\left[\begin{array}{l}
a_{1}(t) \\
a_{2}(t)
\end{array}\right]+d\left[\begin{array}{l}
s_{i 1}(t) \\
s_{i 2}(t)
\end{array}\right]
$$

Obtaining relations for $\omega_{m}$ and $\delta \omega$ is facilitated by transforming to the supermode basis. To that end, Eq. (8) is multiplied from the left by $\mathbf{U}$, where $\mathbf{U}$ is constructed from the eigenvectors of the two-resonator system

$$
\mathbf{U}=\frac{1}{\sqrt{2}}\left[\begin{array}{cc}
1 & 1 \\
1 & -1
\end{array}\right]
$$


and $\mathbf{U} \mathbf{U}^{\dagger}=1$. The result is

$$
\begin{aligned}
\frac{d}{d t}\left[\begin{array}{l}
a_{+}(t) \\
a_{-}(t)
\end{array}\right] & \\
= & {\left[\begin{array}{cc}
-i\left(\omega_{0}+\kappa\right)-\gamma & -i \delta \omega \cos \left(\omega_{m} t\right) \\
-i \delta \omega \cos \left(\omega_{m} t\right) & -i\left(\omega_{0}-\kappa\right)-\gamma
\end{array}\right]\left[\begin{array}{l}
a_{+}(t) \\
a_{-}(t)
\end{array}\right] } \\
& +\frac{d}{\sqrt{2}}\left[\begin{array}{l}
s_{i 1}(t)+s_{i 2}(t) \\
s_{i 1}(t)-s_{i 2}(t)
\end{array}\right],
\end{aligned}
$$

where

$$
\left[\begin{array}{l}
a_{+}(t) \\
a_{-}(t)
\end{array}\right]=\mathbf{U}\left[\begin{array}{l}
a_{1}(t) \\
a_{2}(t)
\end{array}\right]
$$

and the coupling between the even and odd supermodes due to the spatially odd time-dependent perturbation is clear from its location in the off-diagonal positions of the matrix.

The optical excitation is incident in waveguide 1 with $s_{i 2}=0$ and time dependence given by $s_{i 1}(t)=s_{i 1}(\omega) e^{-i \omega t}$. The presence of the $\delta \omega \cos \left(\omega_{m} t\right)$ modulation terms will introduce harmonic frequency components spaced by $\omega_{m}$, so solutions of the form

$$
a_{ \pm}(t)=\sum_{s=-\infty}^{+\infty} a_{s, \pm}(\omega) e^{-i\left(\omega+s \omega_{m}\right) t}
$$

are assumed. The resulting equations for the $s$ components after matching frequency terms are

$$
\begin{gathered}
-\left[i\left(\omega+s \omega_{m}-\omega_{0}-\kappa\right)-\gamma\right] a_{s,+}(\omega) \\
+i \frac{\delta \omega}{2}\left[a_{s+1,-}(\omega)+a_{s-1,-}(\omega)\right] \\
=\frac{d}{\sqrt{2}} s_{i 1}(\omega) \delta_{s 0}, \\
-\left[i\left(\omega+s \omega_{m}-\omega_{0}+\kappa\right)-\gamma\right] a_{s,-}(\omega) \\
+i \frac{\delta \omega}{2}\left[a_{s+1,+}(\omega)+a_{s-1,+}(\omega)\right] \\
=\frac{d}{\sqrt{2}} s_{i 1}(\omega) \delta_{s 0},
\end{gathered}
$$

where $\delta_{s 0}$ is the Kronecker delta. These equations are an infinite set of coupled linear equations for the $a_{s, \pm}$ components. However, in order to obtain usable relations for $\omega_{m}$ and $\delta \omega$, the Fourier series [Eq. (12)] is truncated to keep only the fundamental and first harmonics $s=-1,0,1$. This truncation is an approximation that improves as $\omega_{m}$ increases or $\delta \omega$ decreases relative to $\kappa$ and $\gamma$ [37]. Solving for $a_{0,+}$ and $a_{0,-}$ at $\omega=\omega_{0}$ yields

$$
\begin{aligned}
& a_{0,+}\left(\omega_{0}\right)=\frac{d}{\sqrt{2}} \frac{\omega_{m}^{2}-\kappa^{2}+\gamma^{2}+i 2 \kappa \gamma}{\gamma\left[\omega_{m}^{2}+\kappa^{2}+\gamma^{2}+(\delta \omega)^{2} / 2\right]+i \kappa\left[\omega_{m}^{2}-\kappa^{2}-\gamma^{2}-(\delta \omega)^{2} / 2\right]}, \\
& a_{0,-}\left(\omega_{0}\right)=\frac{d}{\sqrt{2}} \frac{\omega_{m}^{2}-\kappa^{2}+\gamma^{2}-i 2 \kappa \gamma}{\gamma\left[\omega_{m}^{2}+\kappa^{2}+\gamma^{2}+(\delta \omega)^{2} / 2\right]-i \kappa\left[\omega_{m}^{2}-\kappa^{2}-\gamma^{2}-(\delta \omega)^{2} / 2\right]}
\end{aligned}
$$

The proposed device concept hinges on extinguishing the optical output by choosing specific values of $\omega_{m}$ and $\delta \omega$. To that end, values of $\omega_{m}$ and $\delta \omega$ are sought that make $S_{21}\left(\omega_{0}\right)=s_{r 2}\left(\omega_{0}\right) / s_{i 1}\left(\omega_{0}\right)=0$. Since $s_{r 2}\left(\omega_{0}\right)=d a_{2}\left(\omega_{0}\right)=(d / \sqrt{2})\left[a_{0,+}\left(\omega_{0}\right)-a_{0,-}\left(\omega_{0}\right)\right]$, making $a_{0,+}\left(\omega_{0}\right)=a_{0,-}\left(\omega_{0}\right)$ will cause $S_{21}\left(\omega_{0}\right)=0$. After some manipulation of Eqs. (14) and neglecting the imaginary part of $\kappa$, choosing

$$
\delta \omega=\left[2 \frac{\kappa^{2}\left(\omega_{m}^{2}-\kappa^{2}-\gamma^{2}\right)-\omega_{m}^{2}\left(\omega_{m}^{2}-\kappa^{2}+\gamma^{2}\right)-\gamma^{2}\left(\omega_{m}^{2}+\kappa^{2}+\gamma^{2}\right)}{\kappa^{2}+\gamma^{2}-\omega_{m}^{2}}\right]^{1 / 2},
$$

along with $\omega_{m}^{2}>\kappa^{2}+\gamma^{2}$, will force $S_{21}\left(\omega_{0}\right)=0$. 
Figure 4(b) shows the dependence of $\delta \omega$ on $\omega_{m}$ for cavity parameters $\gamma=1.461 \times 10^{11} \mathrm{~s}^{-1}$ and $\kappa=2.70 \times$ $10^{12} \mathrm{~s}^{-1}$. The minimum value of $\omega_{m}$ is $2 \pi(489 \mathrm{GHz})$. The minimum value of $\delta \omega$ and the value of $\omega_{m, \min }$ for which it occurs can be found by differentiating Eq. (15) with respect to $\omega_{m}$ and setting the result equal to zero. The result is

$$
\omega_{m, \min }=\left[\kappa^{2}+\gamma^{2}+2 \gamma \sqrt{\kappa^{2}+\gamma^{2}}\right]^{1 / 2} .
$$

In the present case, the minimum modulation amplitude of $\delta \omega / \omega_{0}=6 \times 10^{-3}$ occurs at $\omega_{m \text {, min }}=2 \pi(683 \mathrm{GHz})$, as shown in Fig. 4(b). Figure 4(c) shows the scattering spectra obtained from CMT where the reduction in $\left|S_{21}\left(\omega_{0}\right)\right|^{2}$ due to the applied modulation is apparent. Figure 4(c) also shows the scattering spectra obtained from 2D FDTD numerical simulation. For the simulation, a $\Delta n$ value of 0.02855 is used to produce the desired $\delta \omega$. The good qualitative agreement between CMT and FDTD validates the design constraints obtained from CMT. When the proposed modulation scheme is applied in the FDTD simulation, $\left|S_{21}\left(\omega_{0}\right)\right|^{2}$ is reduced to $8 \times 10^{-3}$ at $\omega_{0}=2 \pi(193.5 \mathrm{THz})$. When the modulation is turned off, $\left|S_{21}\left(\omega_{0}\right)\right|^{2}=0.582$, as depicted in Fig. 3(d), so applying the modulation swings the output by $18.6 \mathrm{~dB}$. Figure 4(d) depicts the spatial distribution of the magnetic field $H_{z}(x, y)$ for excitation at $\omega_{0}$ when the modulation is on and off calculated via 2D FDTD. It is apparent that the energy in the output waveguide is minimized when the modulation is applied in accordance with Eqs. (15) and (16). Additionally, the energy in cavity 2 is also minimized, consistent with the CMT predictions described above.

In a very general sense, actuating the output power in this way bears some similarity to electromagnetically induced transparency in the sense that it uses two simultaneous excitations at two frequencies [in this case, one optical ("probe") and one microwave ("dressing")] to induce interference between eigenstates of the system [46]. However, in this device, output extinction, rather than transparency, is the objective. More specifically, according to Eq. (11), the condition $a_{0,+}\left(\omega_{0}\right)=a_{0,-}\left(\omega_{0}\right)$ that leads to $\left|S_{21}\left(\omega_{0}\right)\right|^{2}=0$ also leads to $a_{0,2}\left(\omega_{0}\right)=0$. So the modulation scheme couples the eigenstates $a_{0,+}\left(\omega_{0}\right)$ and $a_{0,-}\left(\omega_{0}\right)$ in such a manner as to cancel the energy in resonator 2 . But the energy in resonator 2 only comes from coupling to resonator 1 , so the condition $\omega_{m}^{2}>\kappa^{2}+\gamma^{2}$ makes intuitive sense if interpreted to mean that the engineered energy exchange rate associated with frequency $\omega_{m}$ must be larger than (or dominate over) the "natural" energy exchange rates characterized by $\kappa$ and $\gamma$.

Further insight is obtained by inspecting the spectra in the "on" [Fig. 3(d)] and "off" [Fig. 4(c)] states. As mentioned previously, without index modulation, $a_{+}(\omega)$ and $a_{-}(\omega)$ are resonant at $\omega_{0}+\kappa$ and $\omega_{0}-\kappa$, respectively. When the modulation is applied, $a_{0,+}(\omega)$ and $a_{0,-}(\omega)$ retain their resonances at frequencies $\omega_{0}+\kappa$ and $\omega_{0}-\kappa$, but they also acquire resonance features shifted by $s \omega_{m}$. Figure 5 shows the scattering spectra calculated via CMT using the same cavity parameters as used in Fig. 4(c). The modulation amplitude is also the same as that in Fig. 4(c), and the modulation frequency $\omega_{m 0}$ corresponds to that used in Fig. 4(c). Figures 5(a)-5(e) depict the scattering spectra when the modulation frequency takes on values from 5 down to 1.5 times $\omega_{m 0}$ while keeping $\delta \omega$ constant. When the modulation frequency is set to $\omega_{m}=5 \omega_{m 0}$, as in Fig. 5(a), the spectra in the vicinity of $\omega_{+}$and $\omega_{-}$ bear resemblance to the spectra when the modulation is off [Fig. 4(c)]. Additionally, there are shallower replicas of the double peak resonance features shifted by $\pm 5 \omega_{m 0}$ [there are small peaks in $\left|S_{21}\right|^{2}$ at these frequencies, but they are difficult to see in Fig. 5(a)]. As the modulation frequency is decreased, the resonance features in the shifted replicas become deeper and move closer to $\omega_{+}$and $\omega_{-}$. At the same time, the transmission peaks at $\omega_{+}$and $\omega_{-}$decrease while the transmission features in the shifted replicas grow. Figures 5(a)-5(f) show that the output extinction results from interference between the fundamental resonances at $\omega_{+}$and $\omega_{-}$and their first harmonics shifted by $\pm \omega_{m}$. The proper choice of $\omega_{m}$ (i.e., $\omega_{m}=\omega_{m 0}$ ) produces spectral overlap of these multiple resonances. Specifically, Figs. 5(e) and 5(f) show that the transmission extinction at $\omega_{0}$ results from destructive interference between the transmission peak at $\omega_{+}$and the shifted replica feature at $\omega_{-}+\omega_{m 0}$ and the destructive interference between the transmission peak at $\omega_{-}$and the shifted replica feature at $\omega_{+}-\omega_{m 0}$. Basically, this is a graphical depiction of what Eq. (13) already says: $a_{0,+}(\omega)$ is coupled to the odd supermode through its first-order harmonics $a_{ \pm 1,-}(\omega)$, and $a_{0,-}(\omega)$ is coupled to the even supermode through its firstorder harmonics $a_{ \pm 1,+}(\omega)$. The spectra shown in Fig. 5(a), in which $\omega_{m}$ is significantly detuned from $\omega_{m 0}$, is reminiscent of a basic phase modulation effect. As the resonant features in the first-order harmonics overlap those of the fundamental, destructive interference occurs, resulting in extinction.

Figure 5(f) corresponds to the "off" state $\left(\omega_{m}=\omega_{m 0}\right)$. The residual peaks that appear in this transmission spectrum come from the uncanceled shifted replicas at $\omega_{+}+$ $\omega_{m 0}$ and $\omega_{-}-\omega_{m 0}$. Figures $5(\mathrm{~g})$ and $5(\mathrm{~h})$ show how the spectra evolve as $\omega_{m}$ is reduced below $\omega_{m 0}$. Ultimately, the various cancelations between the eigenstates and their harmonics are no longer balanced, and a peak at $\omega_{0}$ reemerges.

\section{OPTIMIZING THE CAVITY DESIGN}

Now that the basic device functionality has been illustrated, it is shown how the device can be optimized in the 1D PC cavity platform to meet desired spectral shapes or to use desired modulation parameters. First, increasing the optical output amplitude in the "on" state is investigated. 
(a)

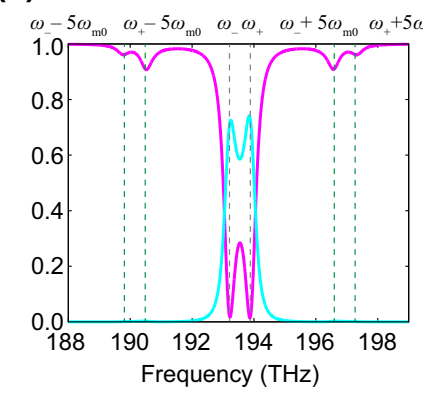

(e)

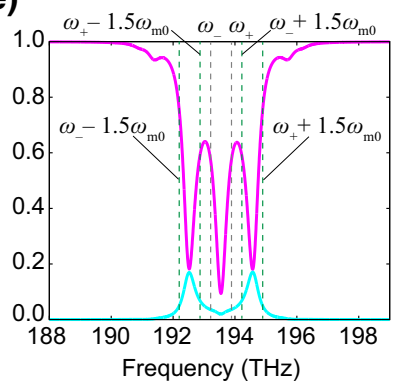

(b)

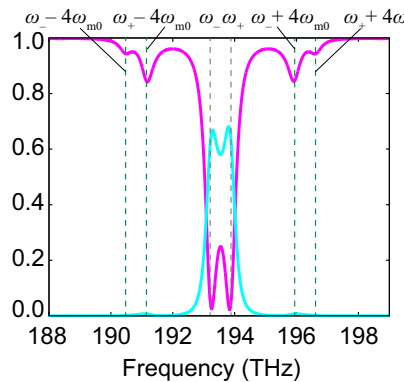

(c)

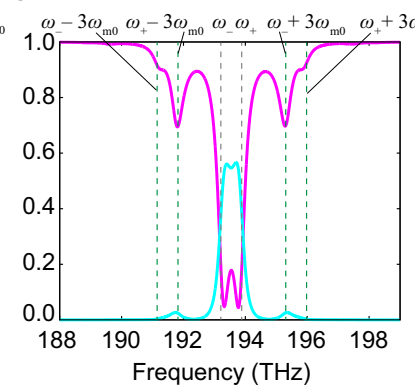

(g)

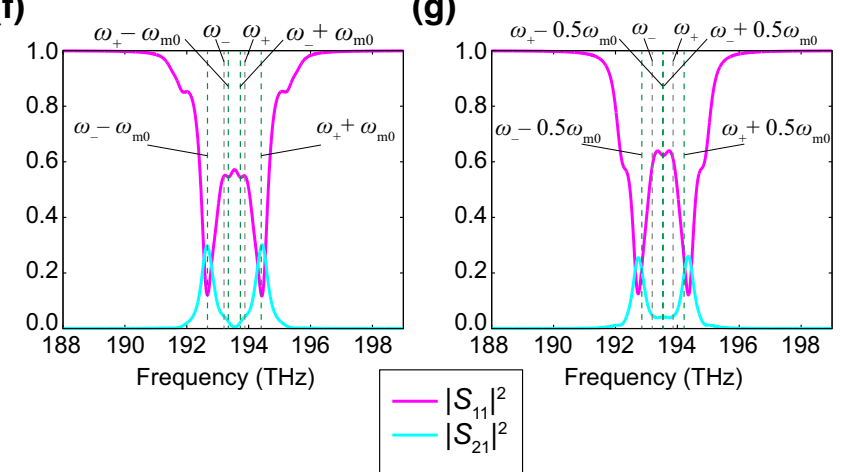

(d)

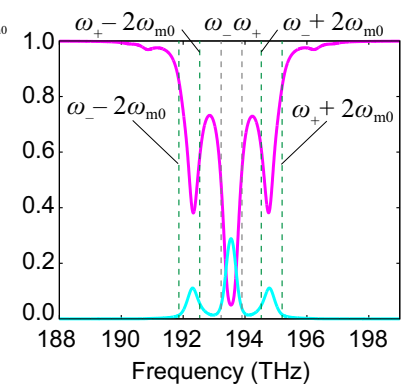

(h)

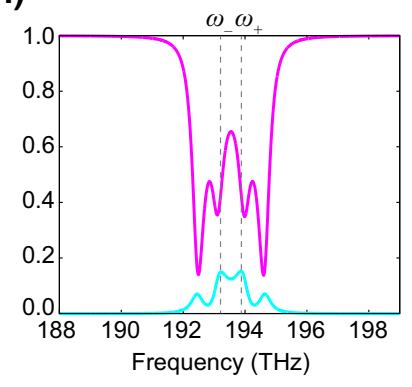

FIG. 5. Scattering spectra calculated using CMT for the same cavity and parameters as Fig. 4. The modulation amplitude is the same as that used in Fig. 4, specifically, $\Delta n=0.02855$. The modulation frequency for producing the "off" state is $\omega_{m 0}=2 \pi(683 \mathrm{GHz})$. Panels (a)-(e) show spectra when the modulation frequency is set larger than $\omega_{m 0}$, with values of (a) $5 \omega_{m 0}$, (b) $4 \omega_{m 0}$, (c) $3 \omega_{m 0}$, (d) $2 \omega_{m 0}$, and (e) $1.5 \omega_{m 0}$. (f) Scattering spectra for the "off" state when $\omega_{m}=\omega_{m 0}$, which is the same as Fig. 4(c). (g) Scattering spectra when $\omega_{m}=0.5 \omega_{m 0}$. (h) Scattering spectra when $\omega_{m}=0.0$.

When the modulation is off,

$$
\left|S_{21}\left(\omega_{0}\right)\right|=\frac{2 \gamma_{c} \kappa}{\gamma^{2}+\kappa^{2}},
$$

where the imaginary part of $\kappa$ is ignored. This function is maximized when $\kappa=\gamma$. Figure 6(a) shows the scattering spectra for different values of $\kappa$, where other cavity parameters are the same as those used in Fig. 3(d). Figure 6(b) confirms the optimal value of $\kappa=\gamma$ that maximizes $\left|S_{21}\left(\omega_{0}\right)\right|^{2}$, which in this case reaches 0.84 . As noted in Fig. 3(b), the number of air holes between the cavities determines $\kappa$ that quantifies the spectral separation between the resonance peaks in the transmission spectrum. Decreasing $\kappa$ by increasing the number of holes between the cavities draws the two resonant peaks closer together. This increases the throughput energy at $\omega_{0}$ when the modulation is off by making the valley between the two peaks shallower. Decreasing $\kappa$ beyond its optimal value causes destructive interference between the two resonance peaks that lowers the throughput at $\omega_{0}$.

From Eq. (17) one sees that increasing $\gamma_{c}$ also increases the throughput when the voltage modulation is off. Figure 6(c) shows the effect on the scattering spectra for different values of $\gamma_{c}$, where other cavity parameters are the same as those in Fig. 3(d). The throughput amplitude increases as expected as does the spectral width of the scattering spectra. As noted in Fig. 3(b), $d$ (and therefore $\gamma_{c}$ ) can be increased by decreasing the number of air holes between the cavities and the input-output waveguides.

In the plots shown in Fig. 6, $\gamma_{0}$ is kept constant at $\gamma_{0}=1.26 \times 10^{11} \mathrm{~s}^{-1}$, so these results should be considered with respect to this value of $\gamma_{0}$. Lossless materials are used in the simulations, so $\gamma_{0}$ is determined only by radiative loss and is related to the cavity quality $(Q)$ factor via $\gamma_{0}=\omega_{0} /(2 Q)$. The value $\gamma_{0}=1.26 \times 10^{11} \mathrm{~s}^{-1}$ corresponds to a $Q$ factor of 4840 . With this value of $\gamma_{0}$ and with $\gamma_{c}=1.34 \times 10^{11}$ and $\kappa=(1.45+i 0.0613) \times 10^{11}$, modulation parameters $f_{m}=510 \mathrm{GHz}$ and $\delta \omega / \omega_{0}=0.0528$ are needed to switch the optical output off. If $\gamma_{0}$ were reduced ( $Q$ increased) by modifying the cavity design, then $\gamma_{c}$ and $\kappa$ could be scaled accordingly using the design concepts discussed above. The scattering spectra would have the same shape but with reduced spectral widths. Because the minimum values of $\omega_{m}$ and $\delta \omega$ are tied to $\kappa$ and $\gamma$, the required modulation parameters also scale with $\gamma_{0}$. Figures 7 (a) and 7(b) show the scattering spectra when $\gamma_{0}$, $\gamma_{c}$, and $\kappa$ are all reduced by a factor of 10 . This corresponds to a higher passive $Q$ factor of $Q=48400$. The spectral shapes are similar to those shown in previous figures but narrower by a factor of 10 . And the modulation parameters used to switch off $\left|S_{21}\left(\omega_{0}\right)\right|^{2}$ are a factor of 10 smaller, $f_{m}=51 \mathrm{GHz}$ and $\delta \omega / \omega_{0}=5.28 \times 10^{-3}$. Of 
(a)

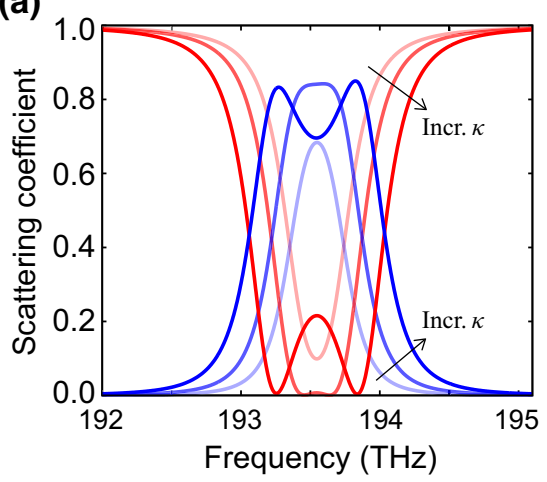

(b)

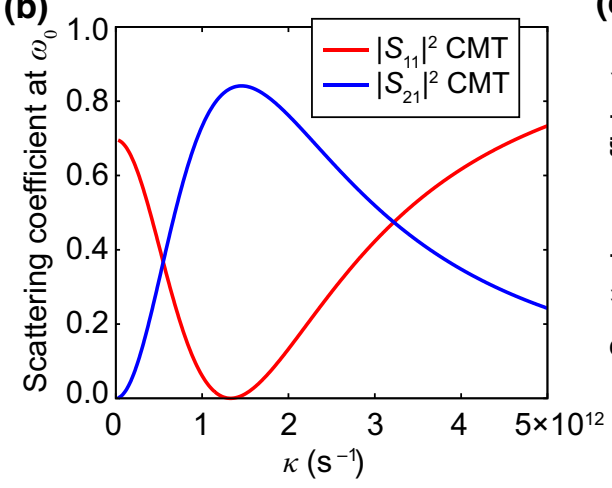

(c)

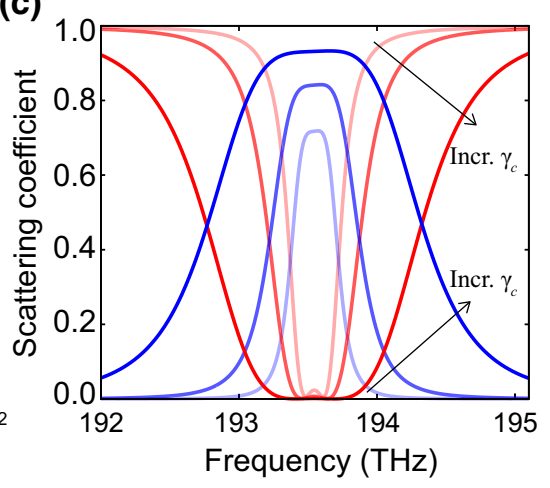

FIG. 6. (a) Scattering spectra for the two-coupled-cavity system shown in Fig. 3(b) with no modulation applied for different values of $\kappa$. Resonator parameters used in the plot are $\omega_{0}=2 \pi(193.5 \mathrm{THz})=2 \pi\left(c_{0} / 1.55 \mu \mathrm{m}\right), \gamma_{0}=1.26 \times 10^{11} \mathrm{~s}^{-1}, \gamma_{c}=1.34 \times 10^{12} \mathrm{~s}^{-1}$. The three $\kappa$ values are $\kappa=(9.17+i 0.0385) \times 10^{11} \mathrm{~s}^{-1}, \kappa=(1.45+i 0.00613) \times 10^{12} \mathrm{~s}^{-1}$, and $\kappa=(2.27+i 0.00955) \times 10^{12} \mathrm{~s}^{-1}$. (b) Plots of $\left|S_{11}\left(\omega_{0}\right)\right|^{2}$ and $\left|S_{21}\left(\omega_{0}\right)\right|^{2}$ as a function of $\kappa$ for the same resonator parameters as in (a). The real part of $\kappa$ is shown on the horizontal axis, but the imaginary part is swept simultaneously while maintaining $\operatorname{Im}[\kappa]=0.00420 \operatorname{Re}[\kappa]$. (c) Scattering spectra for the two-coupled-cavity system shown in Fig. 3(b) with no modulation applied for different values of $\gamma_{c}$. Resonator parameters used in the plot are $\omega_{0}=2 \pi(193.5 \mathrm{THz})=2 \pi\left(c_{0} / 1.55 \mu \mathrm{m}\right), \gamma_{0}=2.00 \times 10^{10} \mathrm{~s}^{-1}, \kappa=(1.45+i 0.00613) \times 10^{11} \mathrm{~s}^{-1}$. The three $\gamma_{c}$ values are $\gamma_{c}=6.72 \times 10^{11} \mathrm{~s}^{-1}, \gamma_{c}=1.34 \times 10^{12} \mathrm{~s}^{-1}$, and $\gamma_{c}=3.35 \times 10^{12} \mathrm{~s}^{-1}$.

particular interest is the lower modulation frequency that is closer to values relevant to communication systems. Ultimately, the modulation frequency can be tuned to any value, assuming that the requisite $Q$ factor can be obtained in a given material platform and cavity design. Figure 7(c) displays the required modulation frequency and modulation amplitude as a function of the $Q$ factor. For operation at the communication frequency $f_{m}=40 \mathrm{GHz}$, a $Q$ factor of $6.2 \times 10^{4}$ or greater and a modulation amplitude of $\delta \omega / \omega_{0}=4.14 \times 10^{-4}$ (corresponding to $\Delta n \approx 1.3 \times 10^{3}$ ) or greater is required. Passive $Q$ factors of this magnitude are routinely demonstrated using modern fabrication capabilities [28]. The requisite $\Delta n$ value is achievable in doped silicon [26] and InP [27].
Though they are not included in the simulations, material losses can be included in the model in a straightforward manner. As mentioned previously, $\gamma_{0}$ is the rate of loss due to all mechanisms other than waveguide coupling; these include radiative losses as well as linear material absorption and scattering. For silicon, reported material losses are in the range of $0.1-1 \mathrm{~dB} / \mathrm{cm}[26,29,31]$, which would decrease the throughput by $10 \%-50 \%$, but this reduction could be mitigated by increasing $\gamma_{c}$ and increasing the drive voltage and frequency concomitantly. InP has larger waveguide losses in the range of $2-3 \mathrm{~dB} / \mathrm{cm}$, placing it at a disadvantage to silicon in terms of loss [30]; however, its amenability to on-chip light sources could, nevertheless, render it useful from a system-wide
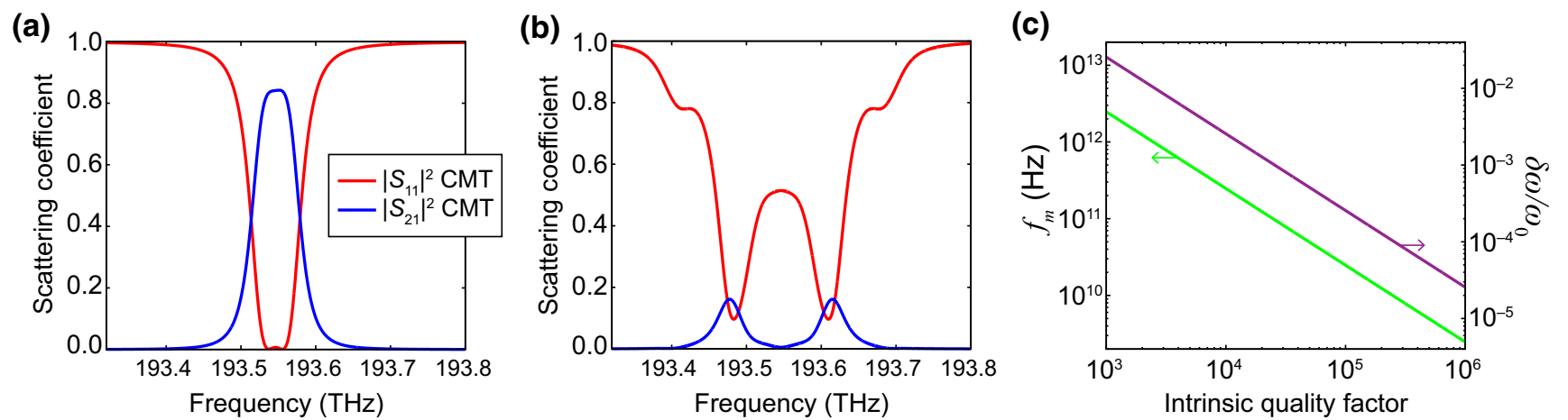

FIG. 7. (a) Scattering spectra for the two-coupled-cavity system with resonance frequency $\omega_{0}=2 \pi(193.5 \mathrm{THz})=2 \pi\left(c_{0} / 1.55 \mu \mathrm{m}\right)$ and loss parameters $\gamma_{0}=1.26 \times 10^{10} \mathrm{~s}^{-1}, \gamma_{c}=1.34 \times 10^{11} \mathrm{~s}^{-1}$, and $\kappa=(1.45+i 0.0613) \times 10^{11} \mathrm{~s}^{-1}$ that are a factor of 10 lower than those used in Fig. 6(a). Here $\kappa$ is chosen to maximize $\left|S_{21}\left(\omega_{0}\right)\right|$ according to Eq. (17) and Fig. 6(b). (b) Scattering spectra when the modulation is applied. Modulation parameters are $f_{m}=51 \mathrm{GHz}$ and $\delta \omega / \omega_{0}=5.28 \times 10^{-3}$. (c) Dependence of $f_{m}$ and $\delta \omega / \omega_{0}$ on the intrinsic quality $(Q)$ factor of the cavity via Eqs. (15) and (16). The quality factor $Q$ is related to $\gamma_{0}$ via $\gamma_{0}=\omega_{0} /(2 Q)$. The ratios $\gamma_{c}=10.6 \gamma_{0}$ and $\operatorname{Re}[\kappa]=\gamma$ are maintained in constructing the plot. Green (purple) line refers to the left (right) axis. 
perspective. Recent advances in the microfabrication of low loss lithium niobate waveguides and resonators makes it a prospective material. Lithium niobate is attractive due to its low absorption and large electro-optic effect $[47,48]$, though tight control of photonic crystal hole size and location could still be challenging to fabricate. The proposed demodulator device could also be implemented in heterogeneous material systems, such as graphene [49], ferroelectric lead zirconate titanate [50], or lithium niobate films [51] on low-loss silicon nitride, which may offer additional design flexibility but are not explicitly designed for here.

In addition to its ability to tune $\kappa$ and $\gamma_{c}$ in a straightforward manner, the 1D PC cavity design offers an ability to tune the $Q$ factor where values of $1.47 \times 10^{5}$ have been experimentally measured [28]. The design is based on reducing radiation loss from the cavity by tapering the size of the holes nearest the cavity. The tapered hole size allows the localized field to extend further along the $x$ direction, thereby making its $k$-space distribution more localized. This reduces $k$-space overlap with the radiation cone defined by total internal reflection at the air-semiconductor interfaces that make up the sides of the ridge waveguide. Ultimately, the extended field along $x$ results in reduced radiation loss along $y$. Using this design methodology, the $Q$ factor of the cavity shown in Fig. 8(a) is increased to $Q=1.69 \times 10^{5}$ by tapering the size of three holes on either side of both cavities. The hole radii are tapered from the nominal size of $r=0.3 w=111.6 \mathrm{~nm}$ to $89.3,55.8$, $39.1 \mathrm{~nm}$. This corresponds to radii scaled by $0.8,0.5$, and 0.35 . The cavity width is reduced to $3.5 w=1.30 \mu \mathrm{m}$ in the optimized version from $4 w=1.49 \mu \mathrm{m}$ in the cavity shown in Fig. 3(b) to counteract the red shift in resonant frequency caused by the increased effective index introduced by the hole taper. A nominal cavity size of $4 w$ corresponds to removing three air holes from a $1 \mathrm{D}$ PC. The number of holes between the two cavities is increased to 14 to obtain the optimal value for $\kappa$.

Figure 8(b) shows the scattering spectra when the modulation is off. At $\omega_{0}$, the power throughput is $\left|S_{21}\left(\omega_{0}\right)\right|^{2}=$ 0.875 . Figure $8(\mathrm{c})$ shows the scattering spectra when the modulation is on with $\Delta n=1.48 \times 10^{-3}$ and $\omega_{m}=$ $2 \pi(23.8 \mathrm{GHz})$. The throughput power is reduced to $\left|S_{21}\left(\omega_{0}\right)\right|^{2}<2 \times 10^{-3}$, which represents a $26.4 \mathrm{~dB}$ swing from on to off. With the increased $Q$ factor of the passive cavity compared to that of the nominal cavity design shown in Fig. 4(a), the magnitude of the modulation parameters required for output extinction is decreased by a similar factor. Figure 8(d) displays the relation between $\omega_{m}$ and $\delta \omega$ determined by Eq. (15). The modulation amplitude is well within realistic parameter ranges for InP and Si [26,27]. The modulation frequency range is consistent with that of practical communication systems operating at $40 \mathrm{GHz}$. Therefore, the device functionality displayed in Fig. 8 is both experimentally feasible and tuned for practical applications.
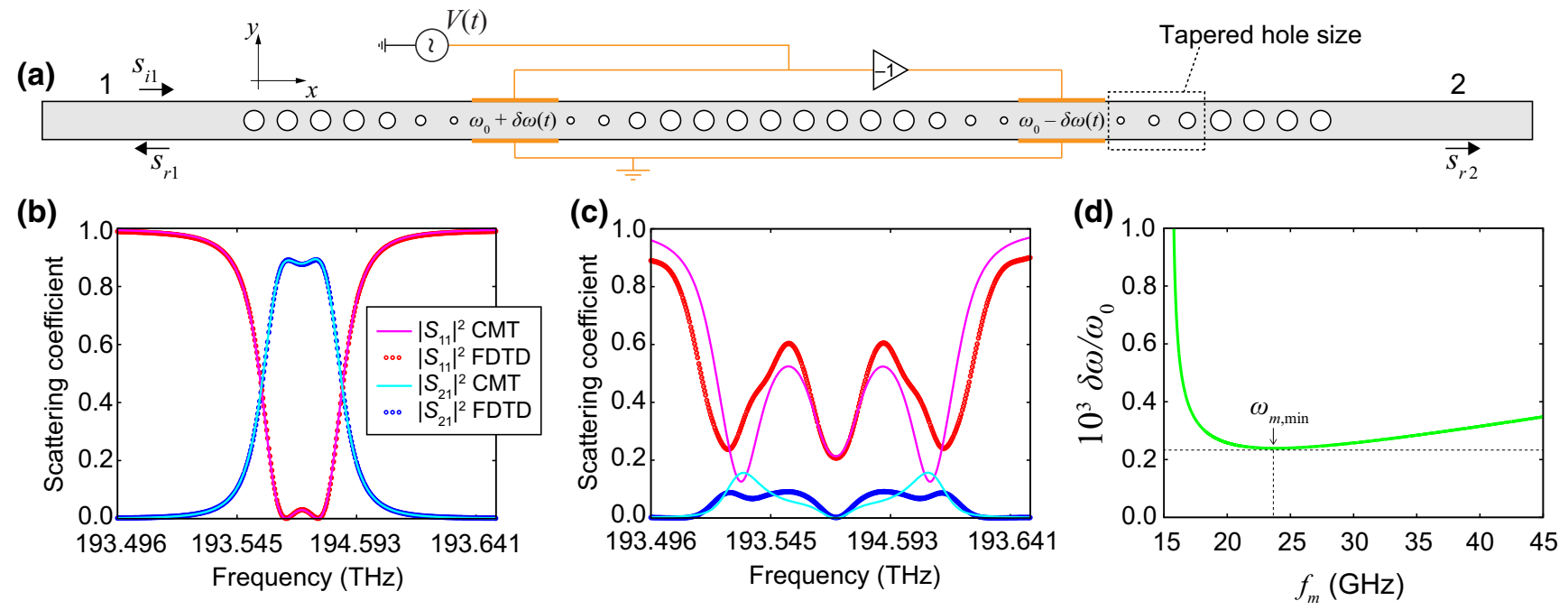

FIG. 8. (a) Cavity design reducing $\gamma_{0}$ by tapering the photonic crystal air hole size on either side of the defect cavities. The three holes adjacent to the cavities are reduced by factors of 0.8, 0.5, and 0.35 compared to the nominal air hole size. The tapered region on the right-hand side of cavity 2 is denoted by a dashed box. Resonator parameters are $\omega_{0}=2 \pi(193.5 \mathrm{THz})=2 \pi\left(c_{0} / 1.55 \mu \mathrm{m}\right)$, $\gamma_{0}=3.59 \times 10^{9} \mathrm{~s}^{-1}, \gamma_{c}=6.13 \times 10^{11} \mathrm{~s}^{-1}$, and $\kappa=(7.35+i 0.0165) \times 10^{10} \mathrm{~s}^{-1}$. This value of $\gamma_{0}$ corresponds to a passive $Q$ factor of 169000 . (b) Scattering spectra for the optimized two-cavity system when the modulation is turned off. (c) Scattering spectra for the optimized two-cavity system when the modulation is turned on, showing the reduction of $\left|S_{21}\left(\omega_{0}\right)\right|^{2}$. The modulation parameters are $\Delta n=0.00148$ and $\omega_{m}=2 \pi(23.8 \mathrm{GHz})$. In both (b) and (c) solid lines are obtained from CMT analysis. Circles are obtained from 2D FDTD simulation. (d) A plot of Eq. (15) for parameters $\gamma=\gamma_{0}+\gamma_{c}$ and $\kappa=7.35 \times 10^{10} \mathrm{~s}^{-1}$. The minimum $\delta \omega$ along with $\omega_{m \text {,min }}$ consistent with Eq. (16) is indicated. 


\section{FREQUENCY CONTROLLED SWITCHING}

Figure 9 shows the dependence of the output scattering amplitude on $f_{m}=\omega_{m} /(2 \pi)$ for different values of $\delta \omega$. The blue curve corresponds to the lowest $\delta \omega$ as determined by Eqs. (15) and (16). As $\omega_{m}$ is increased above the value given by Eq. (16) while keeping $\delta \omega$ unchanged, the output increases from near zero to a value near its value when no modulation is applied at all (approximately 0.875 ). The horizontal dashed line corresponds to half of the maximum value (approximately $0.875 / 2=0.4375$ ), which defines the threshold frequency value for binary symbol determination denoted by $f_{m 0}$. For modulation frequencies above $f_{m 0}$, the output is high, corresponding to a binary one. Likewise, for modulation frequencies below $f_{m 0}$, the output is low, corresponding to a binary zero.

Figure 9 displays another advantageous feature of this device concept, which is its tunability. From the previous analysis, it was found that $\omega_{m}$ can be freely chosen so long as $\omega_{m}^{2}>\kappa^{2}+\gamma^{2}$, and $\delta \omega$ is then set according to Eq. (15). If the modulation amplitude is increased to a larger value, the threshold frequency $f_{m 0}$ increases as well. The red and green curves in Fig. 9 show the dependence of $\left|S_{21}\left(\omega_{0}\right)\right|^{2}$ on $f_{m}=\omega_{m} /(2 \pi)$ for two higher values of $\delta \omega$. In all three cases the same static two-cavity system is used.

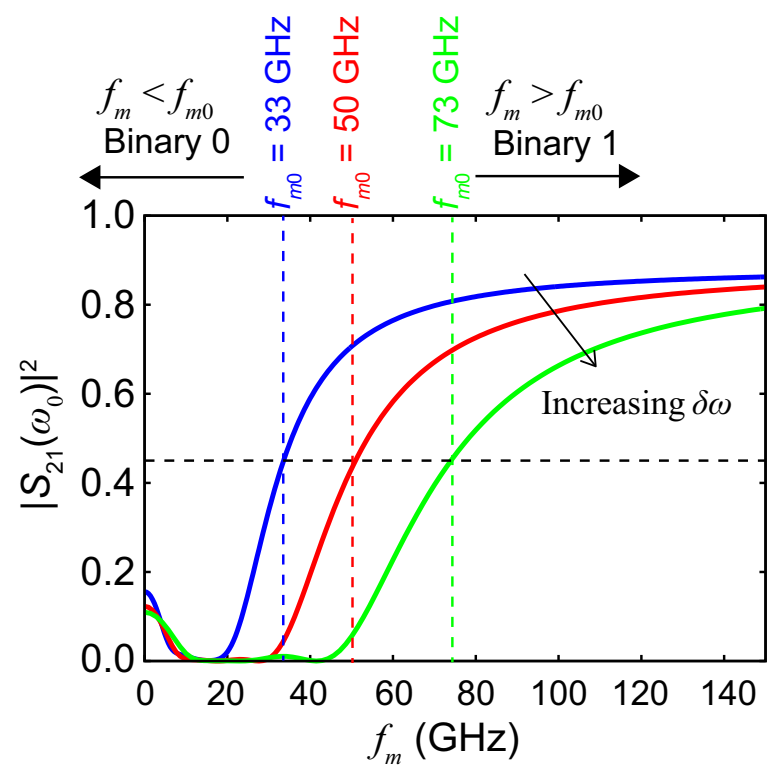

FIG. 9. The dependence of $\left|S_{21}\left(\omega_{0}\right)\right|^{2}$ on $f_{m}=\omega_{m} /(2 \pi)$ for three values of $\delta \omega$. The blue curve corresponds to $\delta \omega / \omega_{0}=$ $1.196 \times 10^{-4}$; the red curve corresponds to $\delta \omega / \omega_{0}=1.825 \times$ $10^{-4}$; the green curve corresponds to $\delta \omega / \omega_{0}=2.658 \times 10^{-4}$. Cavity parameters are $\omega_{0}=2 \pi(193.5 \mathrm{THz})=2 \pi\left(c_{0} / 1.55 \mu \mathrm{m}\right)$, $\gamma_{0}=1.80 \times 10^{9} \mathrm{~s}^{-1}, \gamma_{c}=3.07 \times 10^{11} \mathrm{~s}^{-1}$, and $\kappa=3.68 \times$ $10^{10} \mathrm{~s}^{-1}$. Horizontal dashed line denotes the half-max at 0.4375 . Vertical dashed lines denote the symbol detection frequency thresholds for binary FSK demodulation corresponding to the different values of $\delta \omega$.
This shows how a given fabricated two-cavity system can be used for binary FSK demodulation with symbol detection frequency thresholds in the range $33-73 \mathrm{GHz}$ (and higher).

Returning to the application introduced in Fig. 1 where the device functionality is envisioned to be used to convert a FSK electronic signal into an OOK optical signal that can then be converted to a voltage for direct FSK demodulation or be fed into a radio-over-fiber optical communication system, Fig. 10 displays the time-domain optical output signal $\left|H_{z}(t)\right|^{2}$ from the demodulator shown in Fig. 8 when the frequency of the modulating voltage is switched between $f_{m}=60.0 \mathrm{GHz}$ and $f_{m}=23.8 \mathrm{GHz}$. As noted previously, the frequency $f_{m}=23.8 \mathrm{GHz}$ drives the output to zero as prescribed by Eq. (16) for the cavity design shown in Fig. 8. This produces the "off" state. Detuning the modulation frequency to $f_{m}=60.0 \mathrm{GHz}$ allows the input optical signal to couple to the output waveguide, resulting in the "on" state. Figure 10 illustrates how the FSK signal is converted to an optical ASK output by causing the optical output to switch between on and off states.

In addition to the basic functionality of the proposed demodulator, Fig. 10 illustrates other important temporal features of the device. Figures 10(b) and 10(c) show the response times of the optical output switch as the modulating voltage frequency is changed. Figure 10(b) shows that to switch the optical output from high to low takes $16.8 \mathrm{ps}$. The photon lifetime of the system due to radiation and waveguide coupling is $\tau_{p}=1 / \gamma=15.4 \mathrm{ps}$. That the fall time is approximately equal to $\tau_{p}$ is reasonable since the energy will presumably radiate out of the cavity system via external radiation and coupling to both the input and output waveguides when the output is switched off using the microwave voltage. Figure 10(c) shows that to switch the optical output from low to high takes $51.5 \mathrm{ps}$, which is $3.34 \tau_{p}$. It is hypothesized that the system has a longer rise time because energy must first build up in cavity 2 before it can couple to the output waveguide. Energy in cavity 2 couples from cavity 1 at a rate defined by $\kappa$ with a coupling time of $1 / \kappa=13.6$ ps. Furthermore, energy is entering the system only from waveguide 1 , whereas $\tau_{p}$ considers coupling to both waveguides. With these considerations, an approximate theoretical prediction of the rise time would be $1 /\left(\gamma_{0}+\gamma_{c} / 2\right)+1 / \kappa=42.8 \mathrm{ps}$, a value approaching the observed time of $51.5 \mathrm{ps}$. Ultimately, the longer response time limits the maximum bit rate for the device, which is $1 / 51.5 \mathrm{ps}=19.4$ Gbps. As mentioned in the Introduction, the minimum bit period typically needed in traditional electronic demodulation schemes is $T=1 / f_{d}$, where $f_{d}$ is the difference frequency between the frequencies used for zero and one. In the present analysis, this comes to $T=27.6 \mathrm{ps}$. Therefore, a rise time of $51.5 \mathrm{ps}$ reduces the maximum bit rate achievable in the present device by 1.87 compared to traditional FSK demodulation. Nevertheless, in situations in which at least two full 

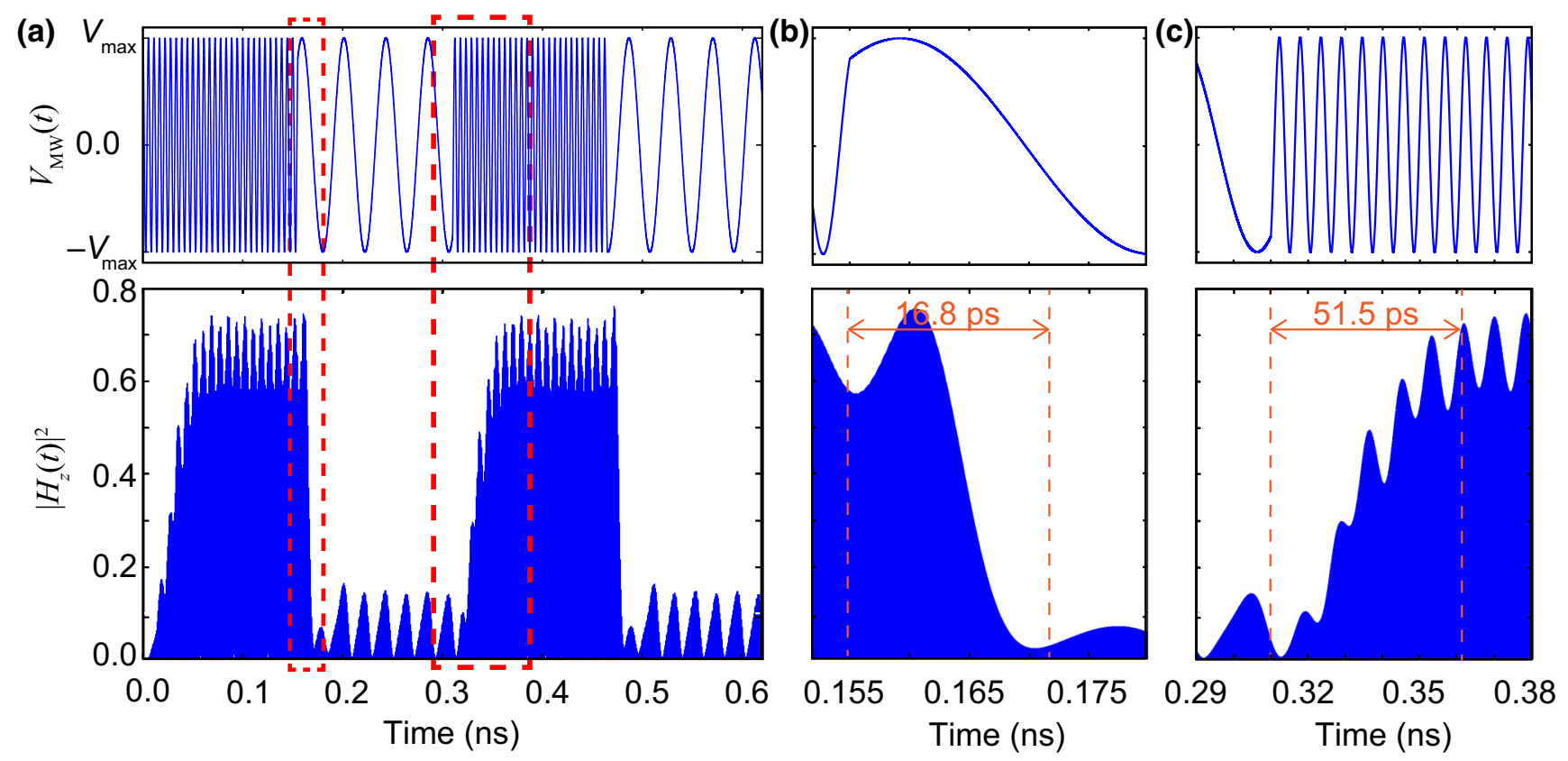

FIG. 10. Top: microwave frequency modulating voltage as a function of time. The frequency switches between $f_{m}=60.0 \mathrm{GHz}$ and $f_{m}=23.8 \mathrm{GHz}$. Bottom: optical output of the demodulator versus time as the applied microwave signal frequency is changed. Here $f_{m}=23.8 \mathrm{GHz}$ corresponds to the off state defined by Eqs. (15) and (16), so the output is low during these frequency intervals. When $f_{m}=60.0 \mathrm{GHz}$, the output is high since Eqs. (15) and (16) are not satisfied by this frequency. (a) Depiction of two on-off cycles of the demodulator. (b) Enlarged view of the first transition from on to off, showing the fall time as 16.8 ps. (c) Enlarged view of a transition from off to on, showing the rise time as $51.5 \mathrm{ps}$.

periods of each microwave waveform for zero and one are used for signaling, the observed rise time would not pose a limitation since two periods of a $23.8 \mathrm{GHz}$ sinusoid is $84.0 \mathrm{ps}$, which is longer than $51.5 \mathrm{ps}$.

\section{REDUCING SIDEBAND ENERGY}

Another feature displayed in Fig. 10 is the presence of ripples both in the "on" and "off" states. Especially in the off state, the ripples can reach to $20 \%$ of the on state amplitude, which significantly reduces the amplitude contrast between on and off. These ripples are due to energy in the modulation sidebands at frequencies $\omega_{0}+s \omega_{m}$ (for nonzero integer $s$ ). Even if the throughput amplitude is driven to zero at $\omega_{0}$, as shown spectrally in Fig. 8(c), there will be energy modulated into sideband components that will ultimately be coupled to the output waveguide. Since this energy is detuned from $\omega_{0}$, one way to remove it is via passive filtering. To this end, Fig. 11 displays how a passive filter with a passband similar to that of the demodulator cavities may be attached to waveguide 2 to remove the ripples. The filter itself has the same geometry as the isolated cavities and is placed a few wavelengths beyond the right cladding of cavity 2 , as shown in the inset of Fig. 11. The plot in Fig. 11 shows the time domain output after passage through the passive filter. The ripples in the off state are significantly reduced, which shows the effectiveness of this strategy to reduce sideband energy.
The on state pulses are reduced in amplitude and less sharp than those shown in Fig. 10, but a more sophisticated filter design could alleviate those issues. Specifically,

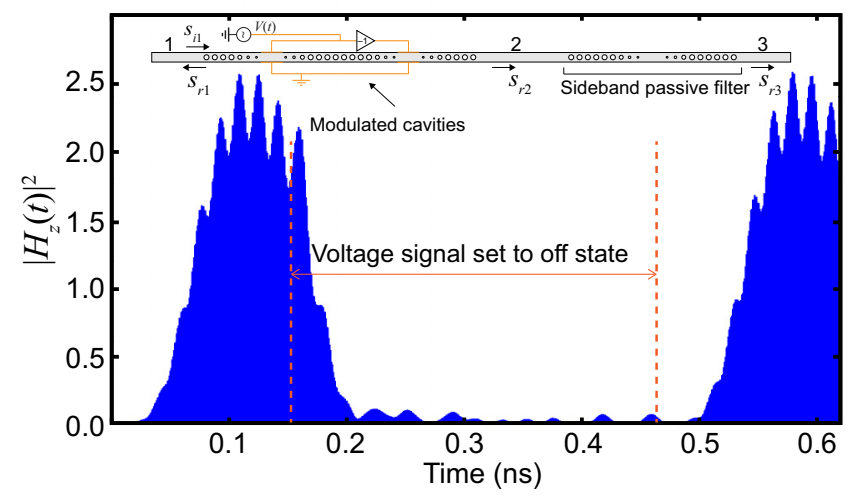

FIG. 11. Optical output of the demodulator shown in Fig. 8 after passing through a passive filter to remove sideband energy. The modulation amplitude is $\Delta n=1.48 \times 10^{-3}$. From 0 to $0.155 \mathrm{~ns}$, the modulation frequency is $\omega_{m}=2 \pi(60.0 \mathrm{GHz})$, which produces the on state. From 0.155 to $0.465 \mathrm{~ns}$, the modulation frequency is set to $\omega_{m}=2 \pi(23.8 \mathrm{GHz})$, which produces the off state. At $0.465 \mathrm{~ns}$, the modulation frequency is set back to $\omega_{m}=2 \pi(60.0 \mathrm{GHz})$ to produce the on state. The inset shows the geometry modeled using FDTD. The filter has the same cavity design as that shown in Fig. 8 except it is a single cavity with five cladding holes beyond the taper region. 
the filter can be optimized to produce a sharper roll off between $\omega_{0}$ and $\omega_{0} \pm \omega_{m}$ to suppress the sidebands more completely [2]. A ring resonator filter could also be attractive for isolating the signal at $\omega_{0}$ while also reducing back reflection not just at $\omega_{0}$ but at $\omega_{0} \pm \omega_{m}$ and higher-order harmonics as well. Nevertheless, as a proof of principle, the time trace shown in Fig. 11 shows the efficacy of a simple passive filter to increase the contrast between the on and off states.

Looking again at Fig. 9, one notes that, as $\delta \omega / \omega_{0}$ is increased, the output port can be switched off for a range of modulation frequencies below that described by Eq. (16). That is, if a $\left(\omega_{m}, \delta \omega\right)$ combination is chosen according to Eqs. (15) and (16), then $\delta \omega$ can be increased while keeping $\omega_{m}$ the same, and the output will remain turned off. For one, this means that the demodulator can be used with a wide range of parameter values. Additionally, Fig. 12(a) shows the output spectrum $\left|S_{21}(\omega)\right|^{2}$ as the modulating voltage amplitude is increased to 1.75 and 2.5 times that described by Eq. (15) while keeping the modulation frequency the same at $f_{m}=23.8 \mathrm{GHz}$. Though the output does not go exactly to zero using these other modulation amplitudes, it remains low nevertheless. Interestingly, it is observed that the range of optical frequencies that are switched off increases as the modulation amplitude increases. Figure 12(d) shows that the CMT predictions are in qualitative agreement with the FDTD modeling even as the modulation amplitude is increased. This suggests a route for dynamic tuning the overall bandwidth of the demodulator.

Additional inspection of Fig. 12(a) reveals that in addition to a widening of the off state bandwidth as $\delta \omega / \omega_{0}$ is increased, the height of the spectral peaks on either wide of $\omega_{0}$ are reduced as well. This offers a potential route toward reducing the ripples caused by sideband energy that were discussed in relation to Fig. 10. Returning to Eqs. (13), it is noted that the sideband energy amplitudes $a_{ \pm 1, \pm}(\omega)$ are related to the fundamental energy amplitudes $a_{0, \pm}(\omega)$ via

$$
\begin{aligned}
a_{ \pm 1,+}(\omega) & =i \frac{\delta \omega}{2} \frac{a_{0,-}(\omega)+a_{ \pm 2,-}(\omega)}{i\left(\omega \pm \omega_{m}-\omega_{0}-\kappa\right)-\gamma} \\
& \approx i \frac{\delta \omega}{2} \frac{a_{0,-}(\omega)}{i\left(\omega \pm \omega_{m}-\omega_{0}-\kappa\right)-\gamma} \\
a_{ \pm 1,-}(\omega) & =i \frac{\delta \omega}{2} \frac{a_{0,+}(\omega)+a_{ \pm 2,+}(\omega)}{i\left(\omega \pm \omega_{m}-\omega_{0}+\kappa\right)-\gamma} \\
& \approx i \frac{\delta \omega}{2} \frac{a_{0,+}(\omega)}{i\left(\omega \pm \omega_{m}-\omega_{0}+\kappa\right)-\gamma}
\end{aligned}
$$

(a)

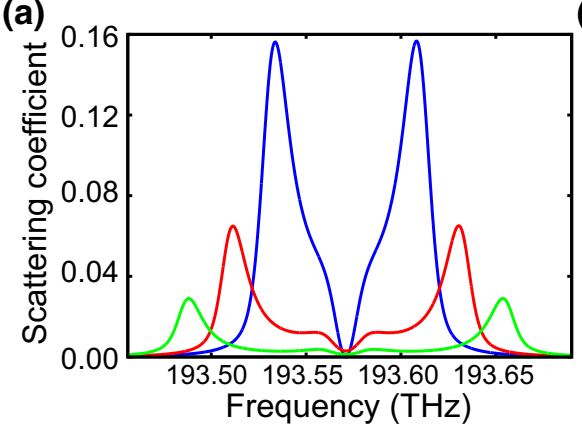

(d)

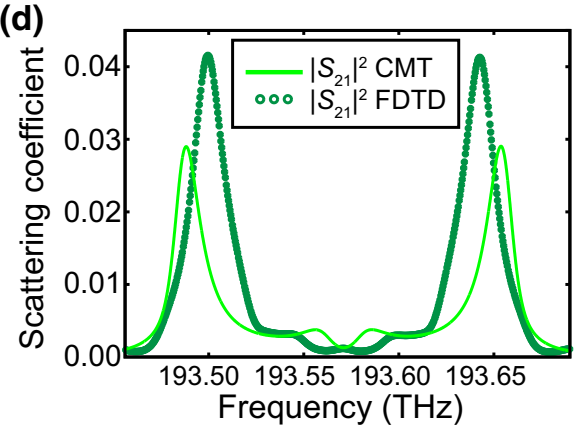

(b)

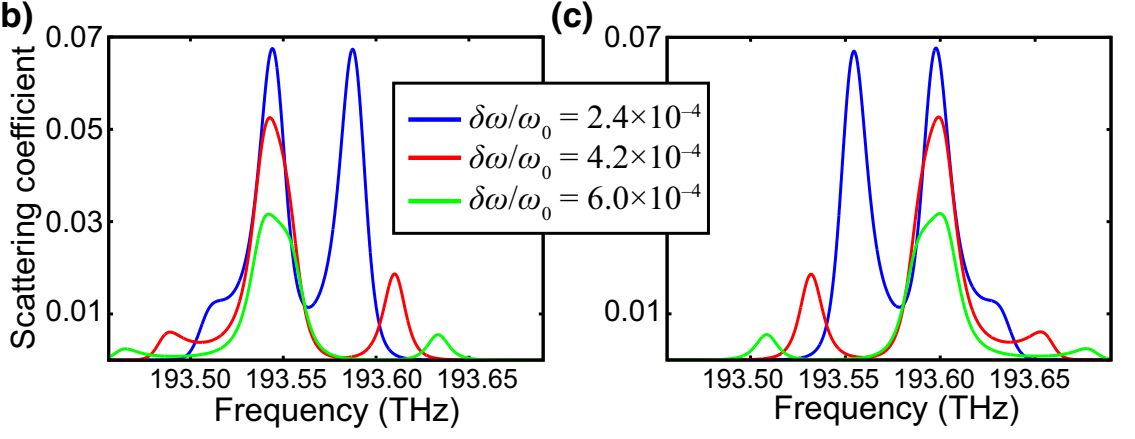

(e)

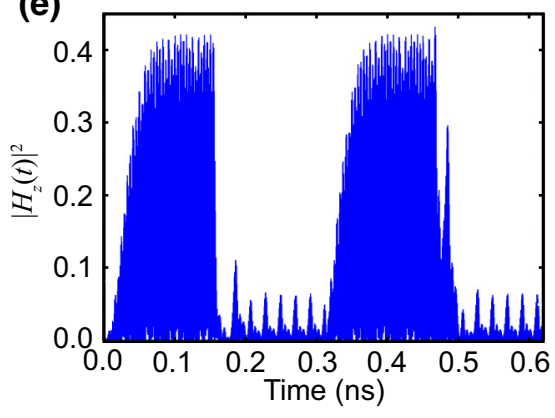

(f)

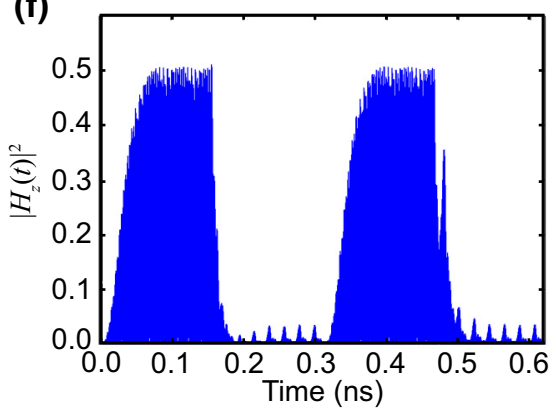

FIG. 12. (a)-(c) Output scattering spectra $\left|S_{21}(\omega)\right|^{2}$ for different voltage modulation amplitudes while keeping the modulation frequency at $f_{m}=23.8 \mathrm{GHz}$. Here $\delta \omega / \omega_{0}=2.4 \times 10^{-4}$ corresponds to the modulation configuration shown in Fig. 8; $\delta \omega / \omega_{0}=$ $4.2 \times 10^{-4}$ and $\delta \omega / \omega_{0}=6.0 \times 10^{-4}$ correspond to 1.75 and 2.5 times larger amplitudes, respectively. The required index change for the three cases is $\Delta n=1.48 \times 10^{-3}, 2.59 \times 10^{-3}$, and $3.70 \times 10^{-3}$, respectively. (a) Depiction of the output spectra at the fundamental frequency. (b) Depiction of the output spectra in the $s=-1$ harmonic. (c) Depiction of the output spectra in the $s=+1$ harmonic. (d) Comparison between CMT and FDTD simulations with $\delta \omega / \omega_{0}=6.0 \times 10^{-4}$. (e) Same as Fig. 10(a) except that $\delta \omega / \omega_{0}=$ $6.0 \times 10^{-4}$, and the "on" frequency is increased to $f_{m}=120 \mathrm{GHz}$. (f) Same as Fig. 10 (a) except that $\delta \omega / \omega_{0}=8.4 \times 10^{-4}$ (3.5 times that in Fig. 8), and the "on" frequency is increased to $f_{m}=180 \mathrm{GHz}$. 
where the approximation neglects harmonics of second order and higher. The amplitude of the output wave resulting from modulation into the first-order harmonics is given by $s_{r 2, \pm 1}(\omega)=(d / \sqrt{2})\left[a_{ \pm 1,+}\left(\omega_{0}\right)-a_{ \pm 1,-}\left(\omega_{0}\right)\right]$, and the corresponding scattering coefficients are defined as $S_{21, \pm 1}(\omega)=s_{r 2, \pm 1}(\omega) / s_{i 1}(\omega)$. This analysis shows the direct proportionality between the wave energy at the fundamental frequency and the wave energy in the first-order harmonics. So if the amplitude of the side peaks is reduced in the fundamental harmonic as shown in Fig. 12(a), then one should expect the amplitudes in the first harmonics to decrease as well. Figures 12(b) and 12(c) show the scattering spectra for the $s=-1$ and $s=+1$ harmonics, respectively, and the decrease in amplitude as $\delta \omega / \omega_{0}$ increases is exhibited. Figures 12(e) and 12(f) show $\left|H_{z}(t)\right|^{2}$ for $\delta \omega / \omega_{0}=6.0 \times 10^{-4}$ and $\delta \omega / \omega_{0}=8.4 \times 10^{-4}$, respectively. In Fig. 12(e), the ratio of ripple height in the off state to the on state amplitude is $15 \%$, which is improved from the nominal ratio of $20 \%$ for the signal depicted in Fig. 10. In Fig. 12(f), the ratio is further decreased to $7 \%$. Though, in both of these cases, a larger detuning frequency is required to reach the on state. Ultimately, this analysis shows how the microwave voltage can be used to dynamically control or suppress the sideband energy in a single fabricated device.

\section{CONCLUSION}

This work presents a frequency demodulator concept based on refractive index modulation of two coupled microcavities excited by an optical carrier wave. An applied microwave frequency voltage changes the refractive indices of the microcavities via the electorefractive effect. The modulating voltage is phase shifted $180^{\circ}$ between the two cavities that induces coupling between the even and odd supermodes. Through CMT and FDTD, it is shown that certain combinations of modulation amplitude and frequency cause the optical output to go to zero at the resonant frequency of the cavities. Required modulation amplitudes and frequencies are tunable and fall within experimentally realizable ranges. As the modulation frequency is detuned from the value prescribed to turn the optical output off, the output turns on and approaches $100 \%$ power transmission for large detuning. This configuration results in amplitude modulation of the optical signal in response to the frequency deviation of an applied microwave voltage. The device functionality is demonstrated through simulation of a high $Q$ factor 1D PC cavity with small footprint. The device can be used for direct frequency demodulation or for multiplexing a FSK microwave voltage into a larger amplitude-modulated radio-over-fiber optical system. For this last application particularly, this demodulator has advantages over existing devices in terms of power, size, and dynamic tunability.
[1] B. E. Little, S. T. Chu, H. A. Haus, J. Foresi, and J.-P. Laine, Microring resonator channel dropping filters, J. Lightwave Technol. 15, 998 (1997).

[2] C. K. Madsen and J. H. Zhao, Optical Filter Design and Analysis: A Signal Processing Approach (Wiley, New York, 1999).

[3] H. Hodaei, M.-A. Miri, M. Heinrich, D. N. Christodoulides, and M. Khajavikhan, Parity-time-symmetric microring lasers, Science 346, 975 (2014).

[4] P. Dong, S. F. Preble, J. T. Robinson, S. Manipatruni, and M. Lipson, Inducing Photonic Transitions between Discrete Modes in a Silicon Optical Microcavity, Phys. Rev. Lett. 100, 033904 (2008).

[5] H. Lira, Z. Yu, S. Fan, and M. Lipson, Electrically Driven Nonreciprocity Induced by Interband Photonic Transition on a Silicon Chip, Phys. Rev. Lett. 109, 033901 (2012).

[6] D. L. Sounas and A. Alù, Non-reciprocal photonics based on time modulation, Nat. Photonics 11, 774 (2017).

[7] Z. Yu and S. Fan, Complete optical isolation created by indirect photonic transitions, Nat. Photonics 3, 91 (2009).

[8] D. L. Sounas and A. Alù, Angular-momentum-biased nanorings to realize magnetic-free integrated optical isolation, ACS Photonics 1, 198 (2014).

[9] A. Zarif, K. Mehrany, M. Memarian, and H. Heydarian, Optical isolation enabled by two time-modulated point perturbations in a ring resonator, Opt. Express 28, 16805 (2020).

[10] A. Mock, D. Sounas, and A. Alù, Magnet-free circulator based on spatiotemporal modulation of photonic crystal defect cavities, ACS Photonics 6, 2056 (2019).

[11] A. Mock, D. L. Sounas, and A. Alù, Tunable Orbital Angular Momentum Radiation from Angular Momentum-Biased Microcavities, Phys. Rev. Lett. 121, 103901 (2018).

[12] J. Capmany and D. Novak, Microwave photonics combines two worlds, Nat. Photonics 1, 319 (2007).

[13] D. Marpaung, J. Yao, and J. Capmany, Integrated microwave photonics, Nat. Photonics 13, 80 (2019).

[14] C. R. Doerr, N. Dupris, and L. Zhang, Optical isolator using two tandem phase modulators, Opt. Lett. 36, 4293 (2011).

[15] Q. Lin, J. Wang, and S. Fan, Compact dynamic optical isolator based on tandem phase modulators, Opt. Lett. 44, 2240 (2019).

[16] J. G. Proakis and M. Salehi, Fundamentals of Communication Systems (Pearson, New Jersey, 2014), 2nd ed.

[17] M. M. Islam, J. Song, K. Rasilainen, and V. Viikari, Optimization of RFID sensor with frequency modulation, IEEE Sens. J. 16, 5993 (2016).

[18] M. Ghovanloo and K. Najafi, A wideband frequency-shift keying wireless link for inductively powered biomedical implants, IEEE Trans. Circuits Syst. 51, 2374 (2004).

[19] E. Main and D. Coffing, An FSK demodulator for bluetooth applications having no external components, IEEE Trans. Circuits Syst. II: Analog and Digit. Signal Process. 49, 373 (2002).

[20] C. Gomez and J. Paradells, Wireless home automation networks: A survey of architectures and technologies, IEEE Commun. Mag. 48, 92 (2010).

[21] Chi-Wai Chow, Ruei-Jie Shiu, Yen-Chun Liu, Xin-Lan Liao, Kun-Hsien Lin, Yi-Chang Wang, and Yi-Yuan Chen, Using advertisement light-panel and CMOS image sensor 
with frequency-shift-keying for visible light communication, Opt. Express 26, 12530 (2018).

[22] A. W. Azim, A. Rullier, Y. L. Guennec, L. Ros, and G. Maury, Energy efficient M-ary frequency-shift keyingbased modulation techniques for visible light communication, IEEE Trans. Cogn. Commun. Netw. 5, 1244 (2019).

[23] M. I. Skolnik, Introduction to Radar Systems (McGrawHill, New York, USA, 2002), 3rd ed.

[24] R. M. Borges, T. R. R. Marins, M. S. B. Cunha, H. R. D. Filgueiras, I. F. da Costa, R. N. da Silva, D. H. Spadoti, L. L. Mendes, and J. Arismar Cerqueira Sodré, Integration of a GFDM-based $5 \mathrm{G}$ transceiver in a GPON using radio over fiber technology, J. Lightwave Technol. 36, 4468 (2018).

[25] Y. Niu, Y. Li, D. Jin, L. Su, and A. V. Vasilakos, A survey of millimeter wave communications (mmWave) for 5G: Opportunities and challenges, Wireless Netw. 21, 2657 (2015).

[26] R. Soref and B. R. Bennett, Electrooptical effects in silicon, IEEE J. Quantum Electron. QE-23, 123 (1987).

[27] B. R. Bennet, R. A. Soref, and J. A. Del Alamo, Carrier-induced change in refractive index of InP. GaAs, and InGaAsP, IEEE J. Quantum Electron. 26, 113 (1990).

[28] A. R. M. Zain, N. P. Johnson, M. Sorel, and R. D. L. Rue, Ultra high quality factor one dimensional photonic crystal/photonic wire micro-cavities in silicon-on-insulator (SOI), Opt. Express 16, 12084 (2008).

[29] Y. Zhang, S. Yang, A. E.-J. Lim, G.-Q. Lo, C. Galland, T. Baehr-Jones, and M. Hochberg, A compact and low loss Y-junction for submicron silicon waveguide, Opt. Express 21, 1310 (2013).

[30] E. Bitincka, G. Gilardi, and M. K. Smi, On-wafer optical loss measurements using ring resonators with integrated sources and detectors, IEEE Photonics J. 6, 6601212 (2010).

[31] M. A. Tran, D. Huang, T. Komljenovic, J. Peters, A. Malik, and J. E. Bowers, Ultra-low-loss silicon waveguides for heterogeneously integrated silicon/III-V photonics, Appl. Sci. 8, 1139 (2018).

[32] M. Burla, C. Hoessbacher, W. Heni, C. Haffner, Y. Fedoryshyn, D. Werner, T. Watanabe, H. Massler, D. L. Elder, L. R. Dalton, and J. Leuthold, $500 \mathrm{GHz}$ plasmonic MachZehnder modulator enabling sub-THz microwave photonics, APL Photonics 4, 056106 (2019).

[33] W. Suh, Z. Wang, and S. Fan, Temporal coupled-mode theory and the presence of non-orthogonal modes in lossless multimode cavities, IEEE J. Quantum Electron. 40, 1511 (2004).

[34] N. A. Estep, D. L. Sounas, J. Soric, and A. Alù, Magneticfree non-reciprocity and isolation based on parametrically modulated coupled-resonator loops, Nat. Phys. 10, 923 (2014).

[35] N. A. Estep, D. L. Sounas, and A. Alù, Magnetless microwave circulators based on spatiotemporally modulated rings of coupled resonators, IEEE Trans. Microw. Theory Tech. 64, 502 (2016).
[36] B. Vial and Y. Hao, A coupling model for quasi-normal modes of photonic resonators, J. Opt. 18, 115004 (2016).

[37] See Supplemental Material http://link.aps.org/supplemen tal/10.1103/PhysRevApplied.15.034056, which includes Ref. [52], for additional details on derivation of Eq. (2) and a discussion of the error in the truncation of Eq. (12).

[38] T. Yang, A. Mock, J. D. O’Brien, S. Lipson, and D. G. Deppe, Lasing characteristics of InAs quantum dot microcavity lasers as a function of temperature and wavelength, Opt. Express 15, 7281 (2007).

[39] M. Nomura, N. Kumagai, S. Iwamoto, Y. Ota, and Y. Arakawa, Photonic crystal nanocavity laser with single quantum dot gain, Opt. Express 17, 15975 (2009).

[40] M. Galli, D. Gerace, K. Welna, T. F. Krauss, L. O’Faolain, G. Guizzetti, and L. C. Andreani, Low-power continuouswave generation of visible harmonics in silicon photonic crystal nanocavities, Opt. Express 18, 26613 (2010).

[41] S. Noda, Photonic crystal lasers-ultimate nanolasers and broad-area coherent lasers, J. Opt. Soc. Am. B 27, B1 (2010).

[42] P. B. Deotare, M. W. McCutcheon, I. W. Frank, M. Khan, and M. Lončar, Coupled photonic crystal nanobeam cavities, Appl. Phys. Lett. 95, 031102 (2009).

[43] B. C. Richards, J. Hendrickson, J. D. Olitzky, R. Gibson, M. Gehl, K. Kieu, U. K. Khankhoje, A. Homyk, A. Scherer, J.-Y. Kim, Y.-H. Lee, G. Khitrova, and H. M. Gibbs, Characterization of 1D photonic crystal nanobeam cavities using curved microfiber, Opt. Express 18, 20558 (2010).

[44] Y. Chen, W. S. Fegadolli, W. M. Jones, A. Scherer, and M. Li, Ultrasensitive gas-phase chemical sensing based on functionalized photonic crystal nanobeam cavities, ACS Nano 8, 522 (2014).

[45] L. Lu, A. Mock, and J. O'Brien, Efficient coupling between a photonic crystal nanocavity and a waveguide with directional end-facet emission, J. Opt. 14, 055502 (2012).

[46] M. Fleischhauer, A. Imamoğlu, and J. P. Marangos, Electromagnetically induced transparency: Optics in coherent media, Rev. Mod. Phys. 77, 633 (2005).

[47] R. Wu, J. Zhang, N. Yao, W. Fang, L. Qiao, Z. Chai, J. Lin, and Y. Cheng, Lithium niobate micro-disk resonators of quality factors above $10^{7}$, Opt. Lett. 43, 4116 (2018).

[48] M. Zhang, C. Wang, R. Cheng, A. Shams-Ansari, and M. Lončar, Monolithic ultra-high- $Q$ lithium niobate microring resonator, Optica 4, 1536 (2018).

[49] C. T. Phare, Y.-H. D. Lee, J. Cardenas, and M. Lipson, Graphene electro-optic modulator with $30 \mathrm{GHz}$ bandwidth, Nat. Photonics 9, 511 (2015).

[50] K. Alexander, J. P. George, J. Verbist, K. Neyts, B. Kuyken, D. V. Thourhout, and J. Beeckman, Nanophotonic Pockels modulators on a silicon nitride platform, Nat. Commun. 9, 3444 (2018).

[51] S. Jin, L. Xu, H. Zhang, and Y. Li, $\mathrm{LiNbO}_{3}$ thin-film modulators using silicon nitride surface ridge waveguides, IEEE Photonics Technol. Lett. 28, 736 (2016).

[52] J. N. Winn, S. Fan, J. D. Joannopoulos, and E. P. Ippen, Interband transitions in photonic crystals, Phys. Rev. B 59, 1551 (1999). 